This item was submitted to Loughborough's Research Repository by the author.

Items in Figshare are protected by copyright, with all rights reserved, unless otherwise indicated.

\title{
ZigZagZ: Improving mechanical performance in extrusion additive manufacturing by nonplanar toolpaths
}

PLEASE CITE THE PUBLISHED VERSION

https://doi.org/10.1016/j.addma.2020.101715

PUBLISHER

Elsevier BV

VERSION

AM (Accepted Manuscript)

\section{PUBLISHER STATEMENT}

This paper was accepted for publication in the journal Additive Manufacturing and the definitive published version is available at https://doi.org/10.1016/j.addma.2020.101715

LICENCE

CC BY-NC-ND 4.0

\section{REPOSITORY RECORD}

Allum, James, Jeremy Kitzinger, Yimeng Li, Vadim Silberschmidt, and Andy Gleadall. 2020. "Zigzagz: Improving Mechanical Performance in Extrusion Additive Manufacturing by Nonplanar Toolpaths". Loughborough University. https://hdl.handle.net/2134/13636583.v1. 


\title{
ZigZagZ: improving mechanical performance in extrusion additive manufacturing by nonplanar toolpaths
}

\author{
James Allum ${ }^{a}$, Jeremy Kitzinger ${ }^{a}$, Yimeng Li ${ }^{b}$, Vadim V. Silberschmidta ${ }^{a}$ Andy Gleadall ${ }^{a}$ \\ a Wolfson School of Mechanical, Electrical and Manufacturing Engineering, Loughborough \\ University, Loughborough, LE11 3TU, UK \\ ${ }^{\mathrm{b}}$ Department of Materials Science and Engineering, City University of Hong Kong, Hong Kong, \\ 83 Tat Chee Avenue, Kowloon 999077, Hong Kong Special Administrative Region, China
}

*Corresponding author - Email: A.Gleadall@Iboro.ac.uk; Tel: +44 (0) 1509227578

\section{Graphical abstract}

\section{ZigZagZ (ZZZ) toolpath development

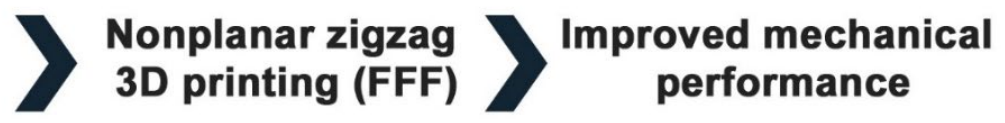

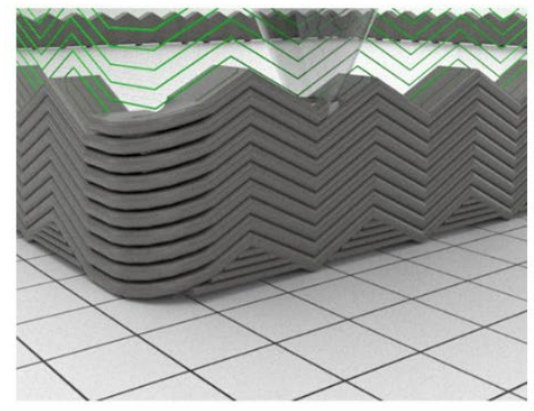
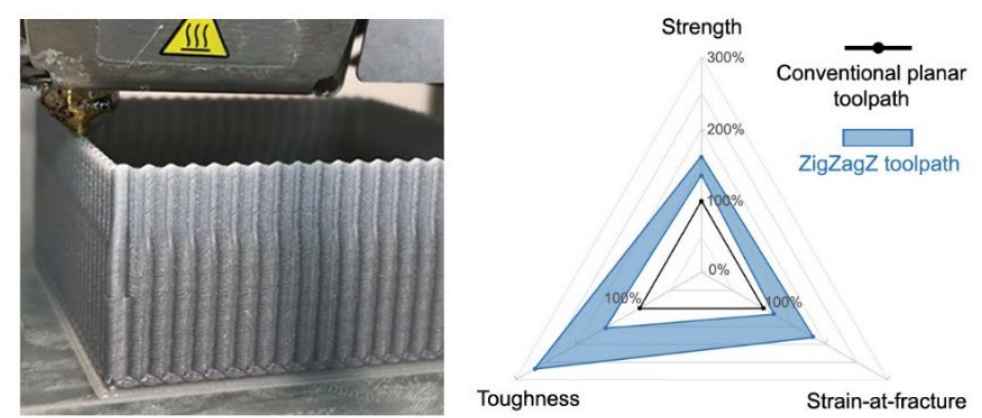

\section{Abstract}

This study investigates the effect of novel non-planar deposition methods in fused filament fabrication (FFF) additive manufacturing. A range of non-planar geometries were developed including a ZigZagZ sequence in which filaments deposited as the nozzle moved in the $X$ or $Y$ direction while simultaneously zigzagging up-and-down i.e. in the $Z$ direction. As a result, repeating non-planar layers were generated throughout the specimen's geometry. The use of the ZigZagZ toolpath to deposit the material significantly improved the mechanical performance of parts manufactured by FFF in the Z-direction by up to $60 \%$ in strength, $119 \%$ in strain-at-fracture and $241 \%$ in toughness compared to an optimised conventional planar geometry. All specimens in the study had only a single filament through their thickness; they were specially developed to enable precise mechanical characterisation. This is the first work to have developed and analysed nonplanar deposition with cyclic nonplanar nozzle movement of a geometric length scale similar to the nozzle diameter. Three novel toolpath designs were developed for this study: (i) zigzag (ZZ), based on the aforementioned ZigZagZ deposition; (ii) up-down (UD) involving vertically deposited nonplanar bulges with interconnecting planar sections; (iii) forward-back (FB) employing the nozzle's movement forward and backward during planar deposition to enhance the nozzle's contact and promote the ploughing of the deposited filament. These designs - along with the conventional planar 
toolpath designs (original (OR)) - were characterised and their mechanical properties compared to generate new understanding about the impact of deposition with the $Z$ coordinate varying along the path on performance. The geometrical outcomes of these different deposition strategies were analysed microscopically to assess the effects of nonplanar toolpaths on the filament-scale geometry. It was established that the ZZ strategies resulted in higher extruded-filament thickness compared to OR. Additionally, various $Z Z$ designs were developed to understand the impact of the zigzag height-to-width ratio and size not only on mechanical properties, but also geometry and fracture path. Fractographic analysis indicated that nonplanar FFF extrusion promoted through-filament fracture, suggesting a reduced concentration of stresses at interlayer bonds by redirecting the load into the filament; this could contribute to the increased level of toughness observed in $\mathbf{Z Z}$ specimens. The understanding developed in this study is readily adaptable for the use in both single-wall and infill geometries to provide their improved mechanical performance. A broad range of potential industrial applications and research relevance resulting from the findings is discussed in addition to future development opportunities.

\section{Nomenclature}

FB - Forward-backward toolpath geometry

FFF - Fused filament fabrication

OR - Original toolpath geometry (conventional planar layers)

PLA - polylactide (polymer)

UD - Up-down toolpath geometry

ZZ - Zigzag toolpath geometry

$Z Z_{D H}$ - Zigzag double height: height dimension of zigzag unit is doubled

ZZDS - Zigzag double size: height and width dimensions of zigzag unit are doubled

$Z_{D W}$ - Zigzag double width: width dimension of zigzag unit is doubled

\section{Introduction}

In recent years, fused filament fabrication (FFF) has become an increasingly popular manufacturing method. The cost effectiveness and accessibility of this technology has supported rapid development and innovation.

In FFF, parts are generated by the extrusion of material (in this study, polymer) through a heated nozzle. A drive motor pushes the material through the nozzle, where it is melted before being deposited onto a platform below. Typically, through the movement of the nozzle, a structure is formed with the thicknesses of a single 'sliced' layer (usually around 0.1 to $0.3 \mathrm{~mm}$ ). Incrementally, at the completion of each layer, the platform is lowered by the height of the layer, enabling the next layer to be manufactured on top of the existing one. This process repeats to build up a part, which is the physical embodiment of the toolpath.

Due to the layer-wise approach in conventional FFF, it is widely acknowledged that the interface between layers is a region of mechanical weakness and cause of mechanical anisotropy. It was reported that incomplete bond formation resulted in reduced material properties at the interface [1-10]. Prior research undertaken by the authors identified that printing-induced geometrical features are rather the cause of diminished mechanical performance $[11,12]$. Additionally, the authors found that interfacial bonding was sufficient to 
give bulk-material strength with the poorer in mechanical performance being attributed to a reduction in the load-bearing area in the region of the interface by filament-scale geometric grooves. These studies concluded that there was a need to overcome these geometrical limitations in order to improve mechanical performance of FFF parts.

This present study is the first to develop a novel nonplanar deposition sequence with nozzlescale nonplanar features. It is based on the zigzag (ZZ) geometry, generated by superposition of the up-and-down movement of the nozzle along the $Z$ direction on the deposition of filaments in the $X-Y$ plane resulting in a geometry formed by repeating nonplanar ZigZagZ features. The purpose of the nonplanar approach is to create a new fundamental building block for FFF 3D-printed parts. The aim of this new geometry is to limit the effect of geometrical features in the region of interface, responsible for reduction of mechanical properties and to generate a unit, which can readily be employed for construction of various geometries irrespective of their overall shape or scale. This present study analyses the extent to which this novel approach addresses and overcomes the mechanical-performance issues identified in the previous studies.

Most studies considered only the parametric modifications, available in 3D printing software such as modifying nozzle temperatures [10,13-16]. Similarly, conventional geometric modifications were predominantly utilised but were typically limited to modifying only the layer height. While layer height reduction was shown in some studies to provide the improved mechanical performance $[10,16,17]$ this was achieved by increasing the ratio of load-bearing material to void and does not directly address the issues of interfacial geometrical features as identified in our studies [11,12].

Less conventionally, one study [18] utilised an alternative deposition strategy to investigate its impact on Z directional performance. The Z-pinning approach generated a grid structure spanning multiple layers. The voids in the grid were subsequently filled, thus resulting in a grid structure with multiple reinforcing 'pins' in the Z-direction. The study concluded that the pinning improved mechanical performance in that direction, but results normalised with functional area ratios indicated that specimens without pins outperformed both solid-fill specimens and pinned specimens in terms of ultimate tensile strength (UTS) in both PLA and carbon-fibre reinforced PLA. Similarly, analysis of an apparent cross-sectional area indicated that the UTS of solid-fill specimens was higher than those with and without pins in both studied materials. Ultimately the study highlighted that investigating the nonconventional 3D printing approaches could lead to new and improved methodologies. Our present study aims to develop an alternative approach to nonplanar deposition that can be employed within structures of all scales and both with and without infill structures.

To the best of the authors' knowledge, the existing experimental studies of non-planar printing examined large, macroscale structures with a focus on either aesthetics or macroscale mechanical performance at the part scale [18-27]. Many of these studies focussed on aesthetic reduction of visible stepping between layers [19,21-25,27] and typically utilised part-scale curved layer approaches [19-21,26,27]. The resultant parts were predominantly mechanically tested in bending [19-22,27]. Our study differs significantly as it considers considerably smaller, nozzle-scale non-planar motions developed to fundamentally innovate the building blocks of the 3D-printed geometry and overcome the filament-scale geometrical limitations identified in the previous studies. So, our concept could be translated to, and utilised in any part geometry irrespective of its scale or 
complexity. This approach also enables the study of the relationship between the nonplanar deposition sequence and the resulting extruded-filament geometry by analysing the effect of the interaction of the nozzle with the deposited material. This insight will allow a better understanding of material deposition and shaping by collisions and troughing at the filament scale.

To date, only a limited number of studies have utilised microscale specimens, with singlefilament thickness $[10,11,28]$. This study utilised a specially designed tensile specimen generated using a custom direct GCode (developed using the freely available FullControl GCode Designer software [29]), which enabled the manufacture of dogbone structures with a wide array of filament geometries, all at the scale of individual filaments. Among the major advantages of this approach is the capability to characterise individual filament and bond interactions at the smallest manufacturing scale (single filaments and the interfaces between them). This allows a fundamental understanding of the geometrical and mechanical performance of the 'building blocks' of FFF by enabling precise analysis without the additional complexities presented by multiple filament interactions. This study is the first to analyse the effect of continuous-toolpath nonplanar printing with features manufactured at nozzle scale for the purpose of improving the mechanical performance between layers in FFF. A wide variety of geometrical factors is considered, including toolpath strategy and filament geometry (extruded-filament thickness also known as raster width) to generate a comprehensive understanding of the geometric influence of the interface.

\section{Materials \& Methodology}

The following section outlines the material and manufacturing methods utilised, including the specially developed single-filament specimens, the method of mechanical characterisation and the toolpath geometry designs.

\subsection{Manufacture of single-filament specimen}

All specimens were manufactured using polylactide (PLA) (Ultimaker branded) with an Ultimaker 2 printer, a $0.4 \mathrm{~mm}$ nozzle diameter was utilised. Nozzle temperature was set at $210{ }^{\circ} \mathrm{C}$, printing at a speed of $25 \mathrm{~mm} / \mathrm{s}$ with a layer height of $0.2 \mathrm{~mm}$. The custom GCode was employed to build four-sided hollow boxes with wall dimensions of $40 \mathrm{~mm}(\mathrm{~L}) \times 45 \mathrm{~mm}$ $(\mathrm{W})$, formed by stacked single filaments with the cross-sectional filament width governing the thickness (Fig. 1). The GCode was created using software developed by the corresponding author (FullControl GCode Designer [29]), which is open-source and freely downloadable from www.fullcontrolgcode.com. It allows explicit definition of the toolpath, which allows planar, nonplanar and other custom printing strategies that are not possible with conventional CAD and slicing methods.

The tensile specimen geometry was specially designed to enable characterisation at the scale of individual filaments, thus reducing the influence of deposition sequence and thermal history, related to manufacturing the parts comprising multiple filaments. Tensile specimens were utilised for mechanical characterisation because interlayer bonds are most susceptible to tensile failure mechanisms. The cross-sectional profile of the walls was produced to replicate a dogbone geometry (Fig. 1), adapted from the micro-tensile specimen of ASTM D1708 - 18 [30]. To prepare the specimens, each box was first cut into four walls, with two 
specimens subsequently cut from each wall using a custom jig, razor blades and a 12-tonne press, resulting in eight specimens per box. The dimensions of each specimen were identical; $40 \mathrm{~mm}(\mathrm{~L}) \times 15 \mathrm{~mm}(\mathrm{~W})$, with a gauge length of $20 \mathrm{~mm}$ (Figs. 1 (b) and (c)). The cross-sectional thickness and geometry were governed by the toolpath geometry and extrusion rate.

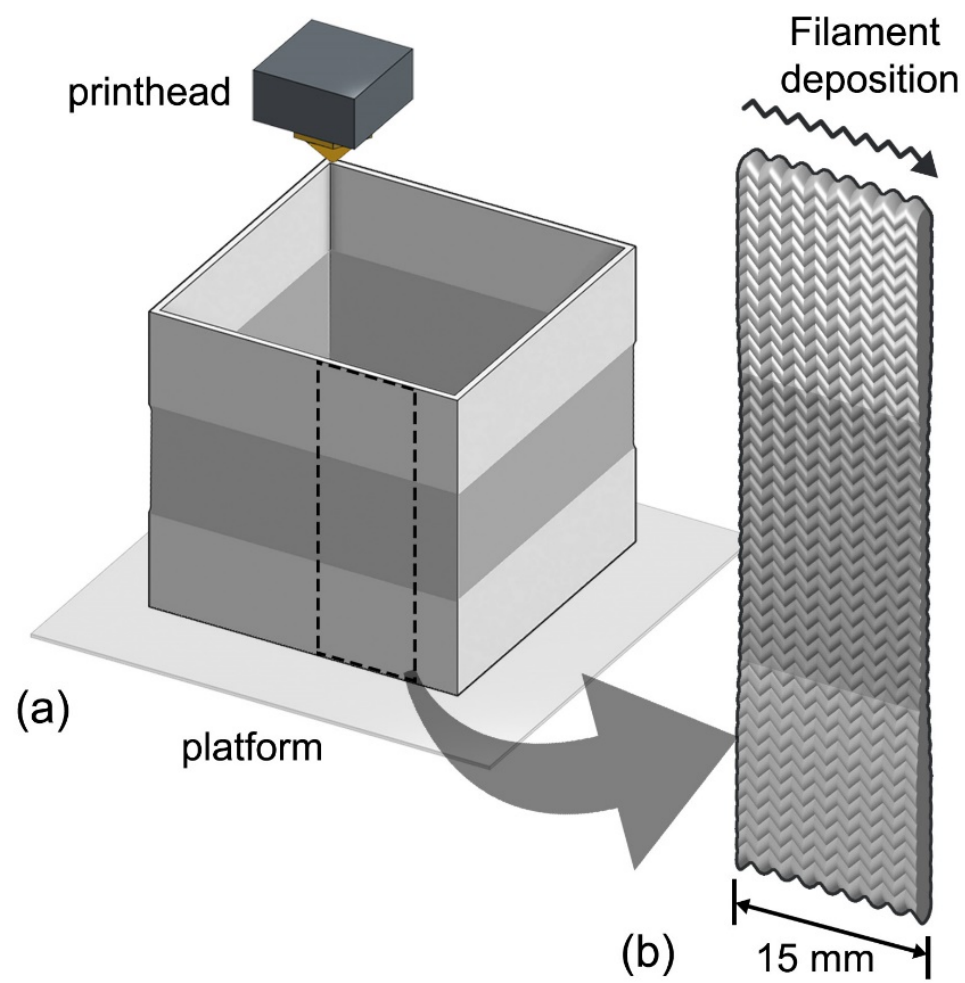

Fig. 1 (a) Manufacture of the box from which specimens (b) are cut and the dimensions of their cross-sectional profile (c).

\subsection{Mechanical and geometrical characterisation}

For each specimen type, five specimens were tested in tension for mechanical characterisation, utilising an Instron 3343 machine equipped with a $1 \mathrm{kN}$ load cell. The extension rate was $0.5 \mathrm{~mm} / \mathrm{min}$. The three remaining specimens were retained for additional measurement. Due to the use of non-planar toolpath, the thickness fluctuated constantly along the cross-section of the specimen gauge, preventing accurate microscopic measurement. Instead, the mean thickness for each specimen type was calculated by weighing a section from the gauge regions of two untested specimens and using the known density of PLA $-1.20 \mathrm{~g} / \mathrm{cm}^{3}$ [31]. In a recent study [32], it was identified that the interfaces between layers have the same properties as the filament, thus, the present study focuses on effects of the geometry of extruded material. To effectively quantify mechanical performance the loading capabilities must be analysed with respect to material usage. Hence, mean thickness of the specimens is used for calculation of mechanical properties. Geometrical characterisation was undertaken with optical microscopy, employing a Zeiss Primotech microscope with $5 x$ magnification lens. 


\subsection{Toolpath design and development}

Four different toolpath geometry types were developed in this study (Fig. 2). Unlike the planar geometry, generated by linear $X-Y$ movement of the nozzle from one corner of the box to the next with a constant $Z$ coordinate, the non-planar geometries were generated by incorporating an intermittent movement of the nozzle in the Z-direction along the toolpath. The resultant non-planar designs were formed by numerous repeating non-planar units with a length of $2 \mathrm{~mm}$.

All specimen types are schematically illustrated with step-by-step toolpath movements in Fig. 2. Irrespective of the toolpath design, each type was generated with a constant filament deposition rate throughout manufacture to alleviate the possibility of introducing defects by the delayed extrusion response identified in another study [33]. The reference planar geometry designated 'original' (OR) (Fig. 2 (a)) was generated by the conventional planar method with filaments deposited unidirectionally in a planar fashion, one layer on top of the next, parallel to the plane of the print platform.

The Zigzag (ZZ) toolpath design (Fig. 2 (b)) was developed with a continuous non-planar sequence with no regions of planar deposition. Filaments were deposited as the nozzle moved in the $X-Y$ plane while simultaneously shifting, first upwards in the $Z$ direction (Fig. 2 (b)(ii)), then downwards (Fig. 2 (b)(iii)), resulting in generation of repeating non-planar zigzag layers throughout the geometry. The sequence of these two steps (Fig. 2 (b)(ii) and (iii)) resulted in the zigzag repeating unit, which, repeated throughout the design formed the overall geometry. A video demonstrating the deposition sequences of the $Z Z$ toolpath geometries is provided as supplementary data (supplementary video S1).

The forward-backward (FB) strategy (Fig. 2 (c)) was developed to analyse the effect of repeated nozzle interaction with the deposited geometry by promoting collisions and nozzle ploughing through bidirectional material deposition without introducing nonplanar movement. The nozzle first deposited material whilst moving in the forward direction in the X-Y plane (Fig. 2 (c)(ii)) before returning half this length in the backward direction (Fig. 2 (c)(iii)), thus creating a repeating-unit sequence comprised of a two steps forward and one step backward. The overall geometry was generated by repeating this sequence (Fig. 2 (c)(ii) and (iii)), throughout. During deposition, each region of the toolpath is interacted with three times (once during the initial forward deposition (Fig. 2 (c)(ii)), secondly during backward deposition (Fig. 2 (c)(iii), and thirdly during the final forward deposition (Fig. 2 (c)(iv)). This approach is focussed on understanding the influence of the nozzle's interaction with the deposited geometry without any nonplanar aspects.

To analyse vertical nonplanar features, the up-down (UD) specimen type was developed (Fig. 2 (d)). UD specimens were produced with planar sections (Fig. 2 (d)(ii)) which linked vertically deposited nonplanar features (Fig. 2 (d)(iii) and (iv)). The vertical features were generated as the nozzle moved, first upwards (Fig. 2 (d) (iii)), then downwards (Fig. 2 (d)(iv)), followed by a length of planar deposition (Fig. 2 (d)(v)). These steps (Fig. 2 (d)(iii) to (v)) formed the UD design unit, which was repeated throughout the design to form the overall specimen geometry. The use of nonplanar and planar features in this design differentiated it from both the conventional planar OR design and the completely nonplanar ZZ design. The UD design enabled geometrical and mechanical analysis of nominally planar layers, but with nonplanar movement of the nozzle applying additional pressure to the layers, enabling a useful comparison with both the conventional planar and nonplanar zigzag strategies. 


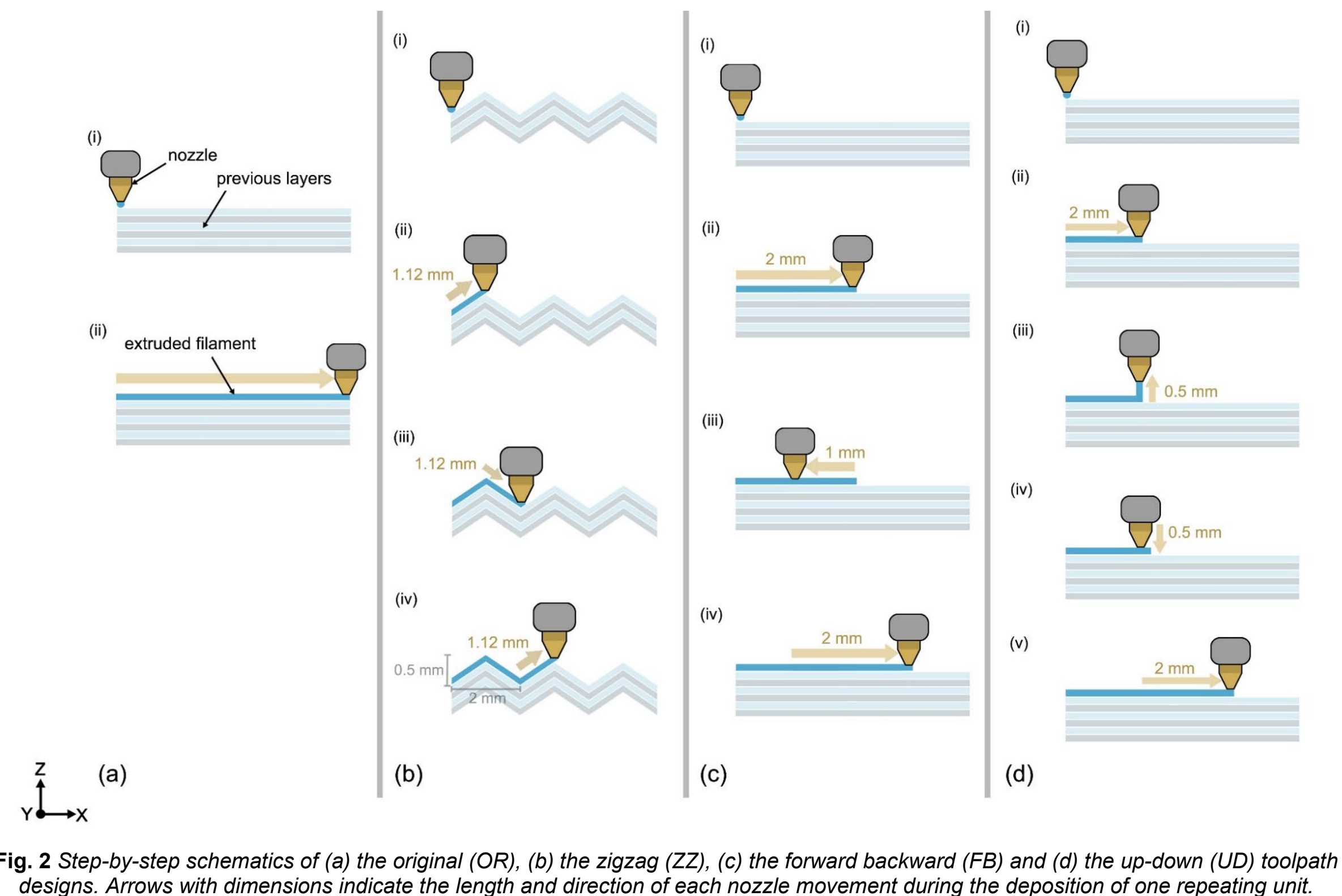

ig. 2 Step-by-step schematics of (a) the original (OR), (b) the zigzag (ZZ), (c) the forward backward (FB) and (d) the up-down (UD) toolpat designs. Arrows with dimensions indicate the length and direction of each nozzle movement during the deposition of one repeating unit. 
A previous study by the authors [12] demonstrated that the extruded-filament geometry (thickness) was an important factor, affecting mechanical performance. Additionally, a preliminary study showed that the nonplanar specimens achieved different thicknesses due to variations in the processing dynamics (discussed in the results section). So, it was decided to generate the $\mathrm{OR}$ and $\mathrm{ZZ}$ specimen types with a range of incrementally increasing thicknesses to investigate its impact on the mechanical performance, the thickness was adjusted by instructing the printer to extrude a greater volume of material (the "E" term in GCode). This was practically achieved in the FullControl design software by specifying the nominal width of extrusions. In addition, several variants of the zigzag geometry were developed to analyse its effect: (i) with double height ( $Z_{\mathrm{DH}}$ ); (ii) with double width (ZZ and (iii) with double height and double width ( $\left.Z Z_{D S}\right)$. Schematics and micrographs of the profiles of the toolpath designs for all $Z Z$ types are presented in Fig. 3, with the printing of the $Z Z_{D S}, Z_{D H}$ and $Z Z_{D W}$ specimens shown in Fig. 4 (a), (b) and (c), respectively. For all $Z Z$ boxes, a support structure was printed at the base (Fig. 4 (d)) to ensure adhesion to the print platform. The video, provided as supplementary data (supplementary video S1),

demonstrates the variation between all $Z Z$ deposition sequences and shows the influence that each of the toolpath designs had on the deposition and resultant geometry.

Due to variations in processing dynamics, specimen thickness was controlled in the range of 0.5 to $1.0 \mathrm{~mm}$ by adjusting the extrusion values in the GCode. The layer height in all specimens was constant at $0.2 \mathrm{~mm}$. 
(a)

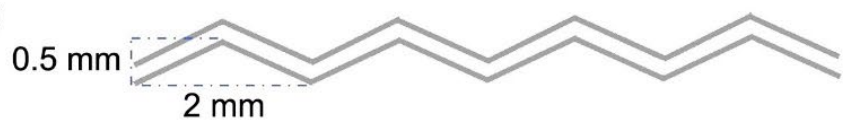

(c)

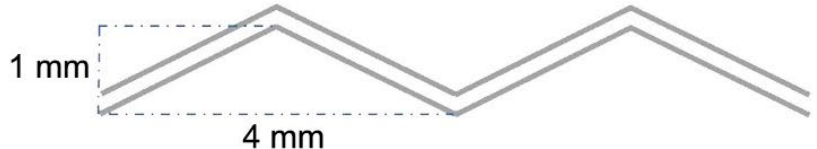

(e)

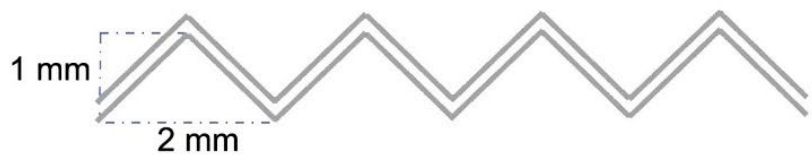

(g)

$0.5 \mathrm{~mm}$

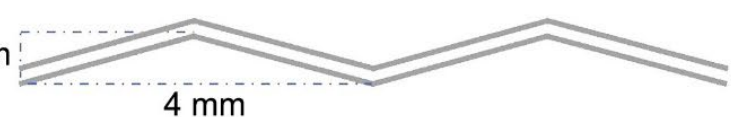

(b)

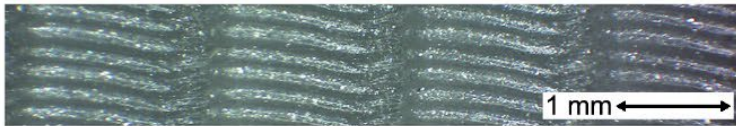

(d)

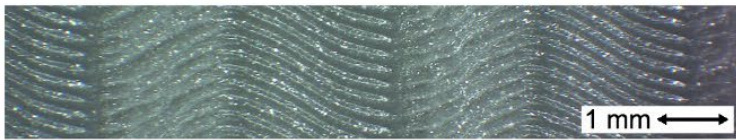

(f)

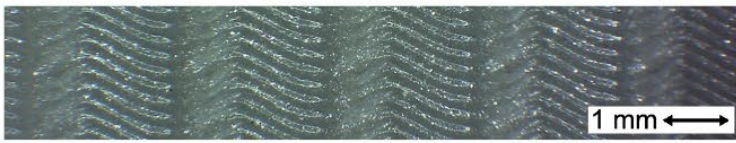

(h)

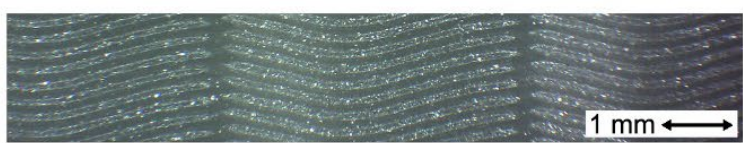

Fig. 3 Schematics (with dimensions) ((a),(c),(e) and (g)) and micrographs ((b), (d), (f) and (h)) of various $Z Z$ specimen types developed in this study: $Z Z$ ((a) and (b)); $Z Z_{D S}\left((c)\right.$ and (d)); $Z Z_{D H}$ $((e)$ and $(f)) ; Z Z_{D W}((g)$ and $(h))$.

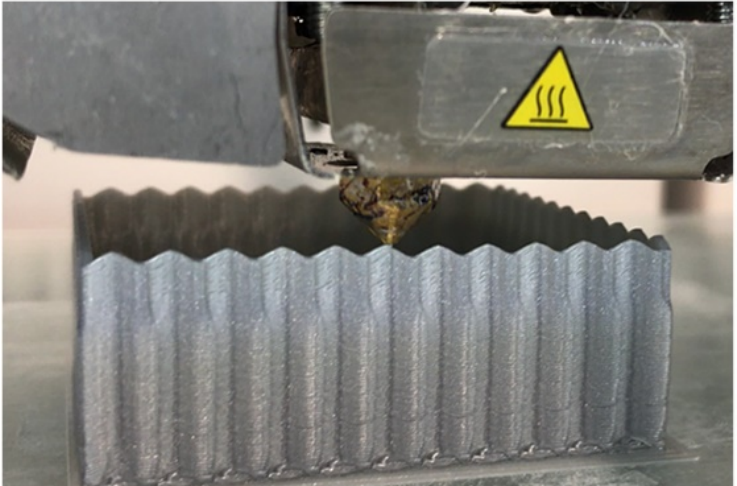

(a)

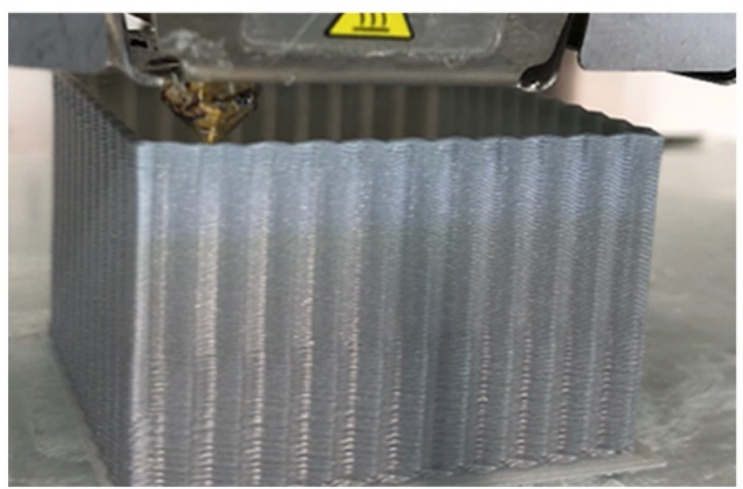

(c)

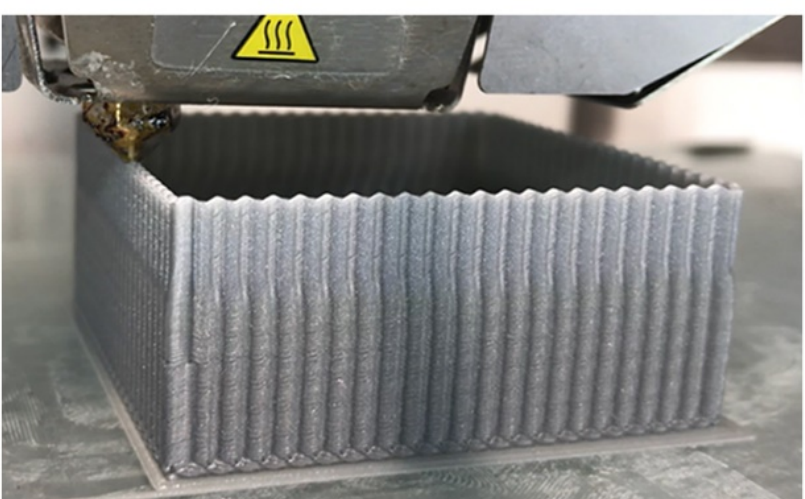

(b)

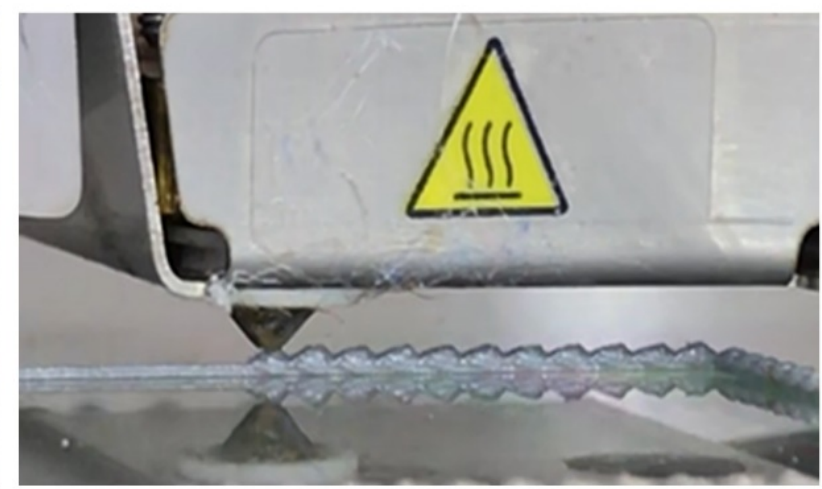

(d)

Fig. 4 Printing processes for generating the $Z Z_{D S}$ (a), $Z Z_{D H}$ (b) and $Z Z_{D W}$ (c) specimens. (d) Support structure for the base of $Z Z$ specimen boxes. 


\section{Results and Discussion}

The results and discussion are divided into the following three sections

- $\quad 3.1$ - Filament geometry, which presents the geometrical characterisation of all specimen types, including dimensional and microscopic analysis.

- 3.2 - Mechanical characterisation, which deals with the mechanical performance of each specimen type including strength, strain-at-fracture and toughness with discussion of the influences that specimen geometry has on performance.

- 3.3 - Fractographic analysis, which studies the fracture paths in each specimen type. Results are discussed with respect to the influence of filament geometry.

\subsection{Filament geometry}

\subsubsection{Filament dimensions}

For each specimen type, the mean thickness of the gauge filaments (Table 1) was calculated by weighing a section of the gauge region of two untested specimens. As demonstrated, four ZZ specimens (denoted ZZ-1 to ZZ-4) had incrementally increasing filament thickness that ranged from $0.45 \mathrm{~mm}$ to $1.07 \mathrm{~mm}$. The filament thickness of OR-1 and OR-2 were similar to that of ZZ-1 and ZZ-2, respectively, enabling direct comparison between them. It was not possible to manufacture wider OR specimens without compromising their mechanical integrity. In any case, the width of OR-2 specimens was more than $150 \%$ of nozzle diameter, i.e. wider than used in almost all research studies and industrial applications. The $\mathrm{ZZ}$ toolpath, however, apparently allowed for an increased rate of extrusion, which is considered in more detail in the next section.

Both UD and FB toolpaths resulted in filament-widths comparable to that of ZZ-3. ZZ and $Z Z_{D W}$ also exhibited the filament thicknesses comparable to those of ZZ-3 and ZZ-4.

This analysis demonstrated that using a conventional 3D-printing system, continuous-printed non-planar strategies could produce filament geometries that not only matched those conventionally achieved in FFF (OR-1 and OR-2), but also exceeded them in terms of filament-width (ZZ-3 and ZZ-4). The next section examines the filament geometry features in more detail to understand the effect of the toolpath designs on the filament-scale geometry. 


\begin{tabular}{|l|c|}
\hline Specimen & Mean filament thickness (mm) \\
\hline OR-1 & 0.49 \\
\hline OR-2 & 0.67 \\
\hline$Z Z-1$ & 0.45 \\
\hline$Z Z-2$ & 0.64 \\
\hline$Z Z-3$ & 0.78 \\
\hline$Z Z-4$ & 1.07 \\
\hline UD & 0.75 \\
\hline FB & 0.72 \\
\hline$Z_{D H}$ & 0.98 \\
\hline$Z_{D S}$ & 0.82 \\
\hline$Z_{D W}$ & 0.73 \\
\hline
\end{tabular}

Table 1 Mean filament thickness $(\mathrm{mm})$ in gauge region of each specimen type.

\subsubsection{Microscopic cross-sectional geometry}

Microscopic analysis of the cross-sections (parallel to the print platform) of all the studied specimen types was undertaken (Fig. 5) to determine the impact of toolpath strategies on the filament-scale geometry; apparently, the strategy had a pronounced effect on this geometry.

OR specimens (Fig. 5 (a) and (b)) demonstrated the common geometrical outcome typical for conventional, planar FFF manufacture: a continuous bead of filament with a constant width and without any other notable geometrical features. As filament thickness was increased, no changes were observed other than the obvious increase in filament thickness.

All ZZ specimens (Fig. 5 (c) - (f) and (i) - (k)) exhibited similar geometrical characteristics. The deposition with the zigzag process resulted in a distinctive structure with a crosssectional geometry (Fig. 6 (a)) that resembled a repeating teardrop pattern. The sequential reference points - (i), (ii) and (iii) (Fig. 6) - indicate various stages in the deposition sequence. Between points (i) and (ii) the nozzle moved in an upwards trajectory, followed by a downward trajectory between points (ii) and (iii) (Fig. 6 (c)) depositing the narrowest filament-width during its upward movement. When moving down, the nozzle acted as a plough, pushing the material forwards and outwards, thus forming the bulge features highlighted in Fig. 6 (b) and (c). Although the toolpath was designed with a symmetrical zigzag profile (shown schematically in Fig. 6 (c)), the bulges resulted in a non-symmetrical profile, as evidenced in the micrograph (Fig. 6 (d)). The formation of the teardrop geometry was in large part due to the ploughing caused by the nozzle, it was not possible to compensate for this bulging by reduction of extrusion rate as the printer was too unresponsive to enable it - an observation which was supported by another study that investigated the responsiveness of extrusion [33].

Changes to the zigzag profile geometry (illustrated in Fig. 3 (a) to (h)) did not prevent the formation of bulges but affected their size and the character of geometric transition. For 
example, this transition was more sudden in $Z_{D H}$ (Fig. 5 (i)), most likely due to the larger angles of ascent and descent in the zigzag, resulting in more material being pushed outwards and, subsequently, a larger bulge. In contrast, the bulging in $Z_{\mathrm{DW}}$ (Fig. $5(\mathrm{k})$ ) was less prominent thanks to smaller angles. $Z_{D S}$ (Fig. 5 (j)) indicated that during the upward phase of zigzag deposition, the filament thickness reduced slightly as the nozzle progressed to the apex of the zigzag. However, more prominently, on the descent, the nozzle produced two bulge-like structures, the second larger than the first generally, it is very likely that the geometry of the zigzag is influenced by the nozzle dimensions; so the features observed in the micrographs (Fig. 5 (c) to (k)) could vary depending on the geometry of the nozzle.

As anticipated, the UD toolpath (Fig. 5 (g)) resulted in repeating bulge features (produced by the up-down motion) linked by a bead of filament with a fairly consistent width (similar to that of the OR toolpath (Fig. 5 ( $a$ and $b)$ ). The bulge in UD was more symmetrical than that in the $\mathrm{ZZ}$ geometries as there was no lateral movement during the vertical ascent or descent movements in this design. Some slight elongation of the bulge was most likely the result of a small amount of material being pushed forward as the nozzle proceeded laterally during the planar movements.

The planar FB toolpath, in which the nozzle moved forwards and backwards over the same section twice, resulted in a fairly consistent and symmetrical geometric outcome (Fig. $5(\mathrm{~h})$ ), with repeating bulge features linked by slightly narrowed regions. These bulges were most likely pulled laterally by the nozzle, producing a more even geometric distribution as compared to the UD design (Fig. $5(\mathrm{~g})$ ). The symmetry of this structure may also have been related to the planar nature of the deposition. The presence of bulging may provide some mechanical benefits to the interlayer region, as these features promote increased crosssectional area and thus are likely to provide increased load-bearing capacity for the Zdirectional loading. 
Main direction of printing

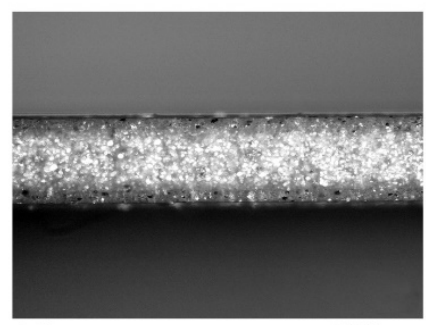

(a)

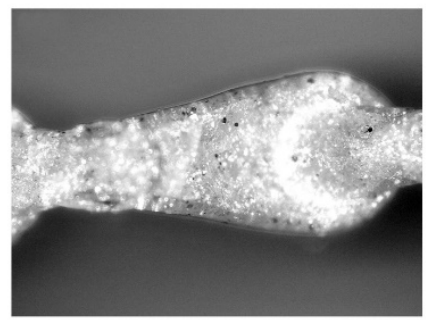

(d)

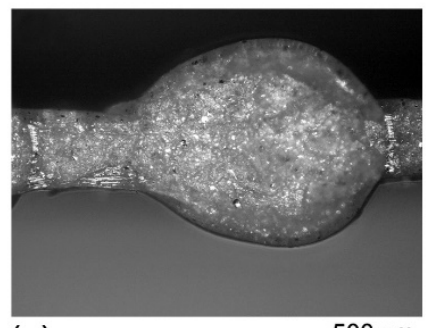

(g)

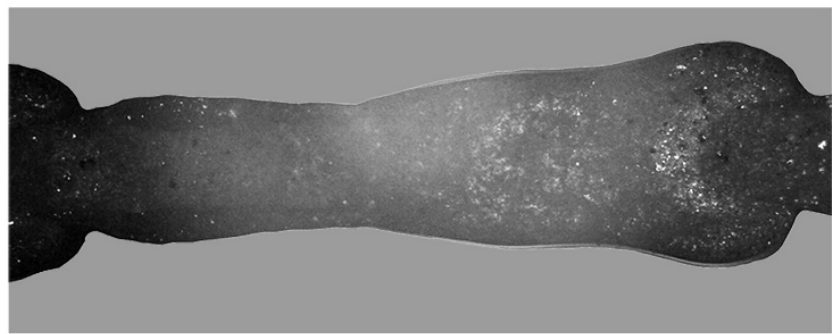

(j)

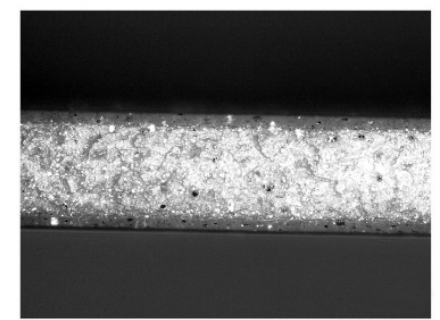

$500 \mu \mathrm{m}$

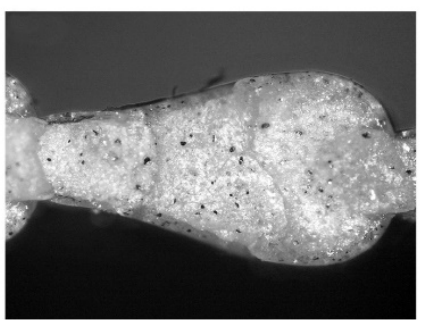

$500 \mu \mathrm{m}$

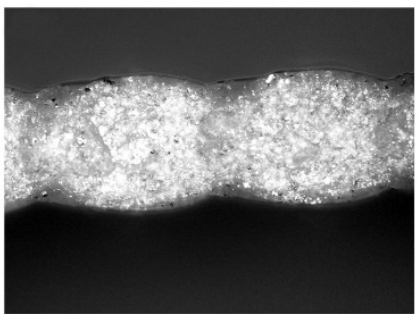

$500 \mu \mathrm{m}$ (i)

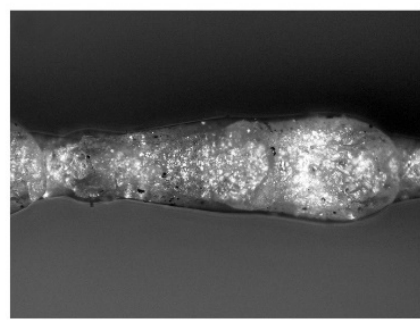

(c)

$\underline{500 \mu \mathrm{m}}$

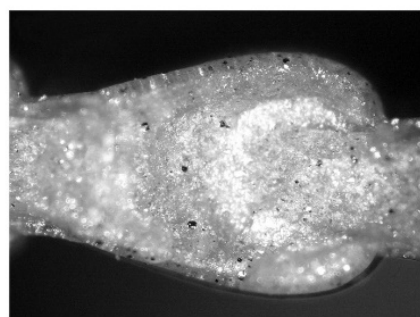

$500 \mu \mathrm{m}$

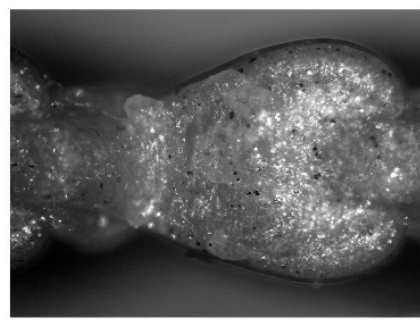

$\underline{500 \mu \mathrm{m}}$

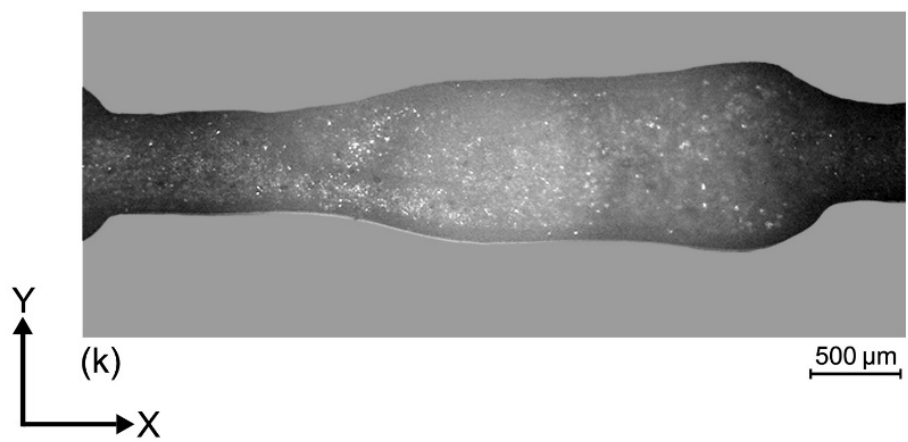

Fig. 5 Cross-sectional geometry of specimens of each type: (a) OR-1; (b) OR-2; (c) ZZ-1; (d) ZZ-2; (e) ZZ-3; (f) ZZ-4; (g) UD; (h) FB; (i) ZZ $Z Z_{D H}$; (j) $Z Z_{D S}$; and (k) $Z Z_{D W}$. 


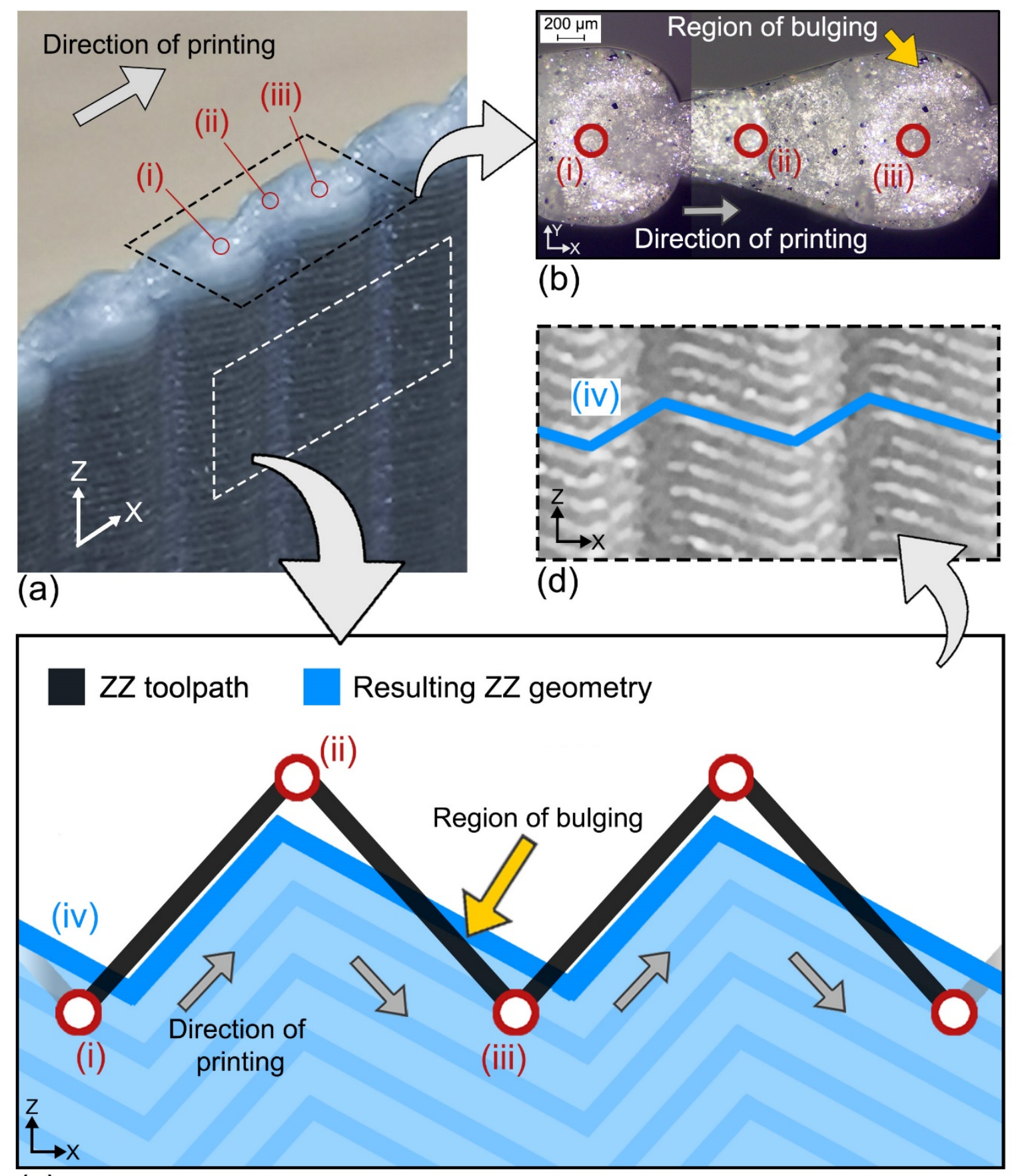

(c)

Fig. 6 Effect of zigzag toolpath on resulting specimen geometry. (a) A closeup view of a specimen with clearly visible regions of bulging as the nozzle moves downward in the $Z$ direction as further detailed in the mircoscopy image (b). Schematic (c) demonstrates the effect of symmetrical movement of the nozzle on the production of asymmetrical features in the resulting geometry with bulging caused by the nozzle's plunging into previously deposited material, forcing it to spread. The closeup side-view image (d) demonstrates the asymmetry of the surface pattern in ZZ specimens due to this zigzag toolpath. 


\subsection{Mechanical characterisation}

Mechanical characterisation of all specimens was undertaken in order to compare the strength, strain-at-fracture and toughness of each specimen type. The study was divided into three parts each focussing on different aspects of the geometry and parameter modification: (i) planar versus non-planar toolpaths (Section 3.2.1); (ii) increased thickness and novel planar versus non-planar zigzag toolpaths (Section 3.2.2); (iii) the zigzag geometry modifications (Section 3.2.3). Each section provides a new comprehension of the effects of filament geometry and toolpath modification on FFF specimens.

\subsubsection{Planar versus non-planar zigzag toolpaths}

To evaluate the effects of planar and nonplanar toolpath geometries, specimens with conventional planar geometries (OR) and with zigzag, nonplanar geometries (ZZ) were considered. In each case, specimens were manufactured in narrow (OR-1 and ZZ-1) and wider (OR-2 and ZZ-2) variants, to examine the effect of filament thickness on the mechanical performance. In each case, the mean filament thicknesses were very close: OR1 and ZZ-1 had mean filament thickness of 0.49 and $0.45 \mathrm{~mm}$, respectively, while OR-2 and ZZ-2 0.67 and $0.64 \mathrm{~mm}$, respectively.

In the case of the two narrower specimen variants, OR-1 and ZZ-1, the specimens demonstrated some differences in mechanical performance (Fig. 7), with the conventional toolpath (OR-1) outperforming the nonplanar (ZZ-1) in terms of the mean effective strength (30.2 MPa versus 24.4 MPa) (Fig. 7 (a)), strain-at-fracture (0.028 versus 0.025$)$ (Fig. 7 (b)) and toughness $\left(0.56 \mathrm{~J} \mathrm{~mm}^{-3}\right.$ versus $0.42 \mathrm{~J} \mathrm{~mm}^{-3}$ ) (Fig. 7 (c)).

However, the opposite trend was observed when the extruded filament thickness was increased as observed for OR-2 and ZZ-2. The zigzag toolpath (ZZ-2) demonstrated a significantly improved mechanical performance compared to the conventional (OR-2), with a mean effective strength of $39.8 \mathrm{MPa}$ versus $29.3 \mathrm{MPa}$, a strain-at-fracture of 0.037 compared to 0.031 , in addition to improved toughness $\left(0.88 \mathrm{~J} \mathrm{~mm}^{-3}\right.$ versus $\left.0.56 \mathrm{~J} \mathrm{~mm}^{-3}\right)$. Additionally, the mean value attained by ZZ-2 specimens was found to be greater than the range of results for OR-2 specimens, with no overlap in the strength, strain-at-fracture or toughness properties (Fig. 8).

Increasing the filament thickness in the conventional toolpath (OR) had little impact on the mechanical performance: increasing the nominal extruded-filament thickness from $0.5 \mathrm{~mm}$ to $0.7 \mathrm{~mm}$ resulted in a $3 \%$ reduction in strength, a $10 \%$ increase in strain-at-fracture, and no significant change in toughness. In contrast, for the zigzag nonplanar geometry, increasing the nominal filament thickness caused the growth of strength by $48 \%$, the strain-at-fracture by $38.7 \%$ and toughness by $70.8 \%$.

These results indicate that conventional specimens reached a plateau in mechanical performance due to geometrical limitations, while the nonplanar specimens (zigzags) showed significant improvements of their mechanical performance as the filament thickness was increased. To understand this further, it was necessary to consider the impact of the increased filament thickness of zigzag specimens on mechanical performance. Additionally, further understanding of the effect of the toolpath on the subsequent geometry (layer time and interlayer adhesion pressure) can be attained. This is the focus of Section 3.2.2. 

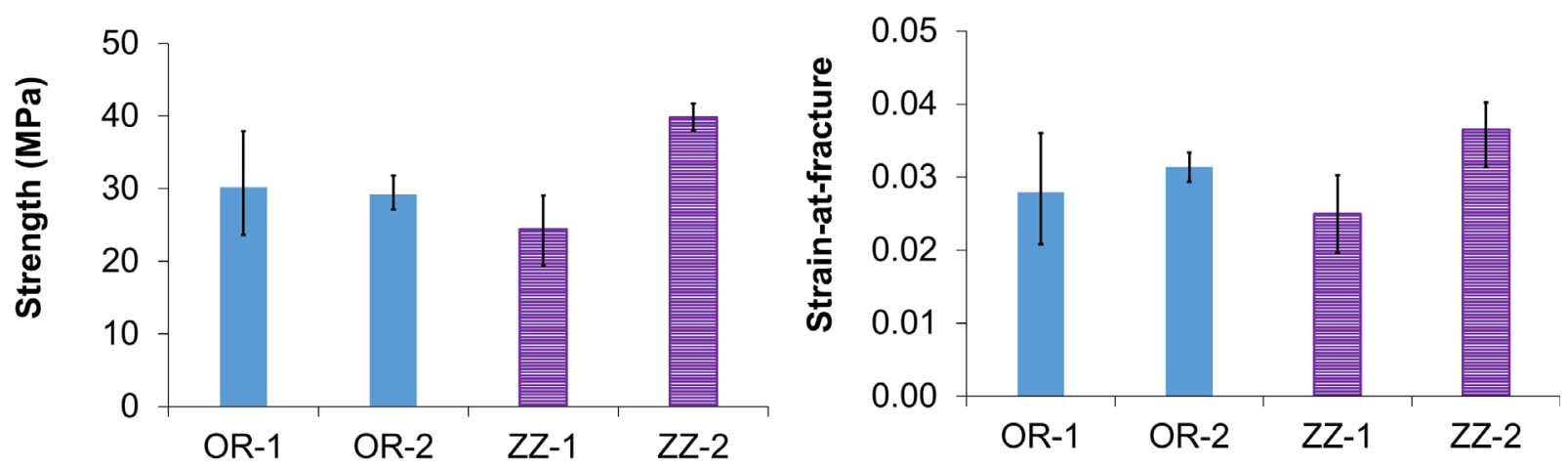

(a)

Specimen type

(b)

Specimen type

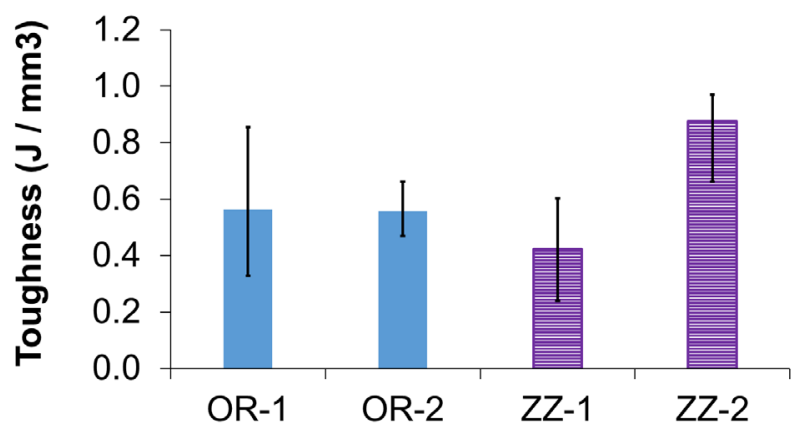

(c)

Specimen type

Fig. 7 Mean effective ultimate tensile strength (a), strain-at-fracture (b) and toughness (c) of OR-1, OR-2, ZZ-1 and ZZ-2 specimens. Error bars indicate the range of results.

\subsubsection{Increased thickness and novel planar versus non-planar zigzag toolpaths}

As demonstrated in the previous section, increasing the filament thickness of the $\mathrm{ZZ}$ specimens improved their mechanical performance beyond that of the equivalent conventional OR specimens. To further understand this effect, the width was further increased in ZZ-3 and ZZ-4 specimens (mean filament thicknesses of 0.78 and $1.07 \mathrm{~mm}$, respectively).

In addition, to understand better performance of these geometries compared to the conventional OR counterparts, two additional geometries were considered: (FB) and (UD). They allowed consideration of increased layer times (FB) and interplane filament deposition (UD) on the mechanical performance. The FB and UD specimens had mean filament thicknesses of 0.72 and $0.75 \mathrm{~mm}$, respectively, close to that of ZZ-3 $(0.78 \mathrm{~mm})$. As discussed, an OR specimen with such thickness had poor mechanical integrity and were not considered. In any case, there was no evidence of improved performance for increasing width in OR specimens.

The mean effective strengths, strain-at-fracture and toughness results for ZZ-3, ZZ-4, FB and UD specimens are given in Fig. 8. ZZ-3 demonstrated improved mechanical performance compared to the ZZ-2 specimen, effective strength increased to $47.5 \mathrm{MPa}$ 
(19.3\% increase). Similarly, strain-at-fracture and toughness also increased to 0.037 and $1.51 \mathrm{~J} \mathrm{~mm}^{-3}$, an increase of $54.1 \%$ and $71.6 \%$, respectively. There was little difference in the effective strength of ZZ-4 compared to ZZ-3; apparently, it reached a plateau, which indicates that the strength was equivalent to bulk-material performance according to the data sheet for the filament (45.6 MPa) [34]. Interestingly, ZZ-4 demonstrated an increase in strain-at-fracture and toughness to 0.069 and $1.92 \mathrm{~J} \mathrm{~mm}^{-3}$, an increase of $86.5 \%$ and $27.2 \%$, respectively, compared to the ZZ-3 specimens. In all instances, ZZ-3 and ZZ-4 significantly outperformed the mechanical behaviour of ZZ-2 specimens. Compared to the OR-2 specimens, the ZZ-3 and ZZ-4 specimens increased in mean effective strength by $62.4 \%$ and $58.5 \%$, strain-at-fracture by $83.9 \%$ and $122.6 \%$, and toughness by $171.8 \%$ and $245.2 \%$, respectively, indicating that the $\mathrm{ZZ}$ geometry resulted in considerable mechanical improvements. Additionally, the mean value of OR-2 specimens was found to be lower than the range of mechanical performance values for ZZ-3, ZZ-4, FB and UD specimens for strength, strain-at-fracture and toughness (Fig. 8).

UD and FB demonstrated similar mechanical performance across all properties: effective strength (41.1 and 40.25 MPa), strain-at-fracture (0.046 and 0.042) and toughness (1.10 and $1.04 \mathrm{~J} \mathrm{~mm}^{-3}$ ). Both UD and FB showed greater mechanical performance compared to the OR specimens, but lower compared to ZZ-3 and ZZ-4. The improvement by UD and FB compared to the OR specimens demonstrates the possibilities to enhance interlayer performance through combinations of: (i) increased thermal exposure and repeated heating when the nozzle moves over the same region multiple times; (ii) polymer mixing by the nozzle thanks to its crushing/bulging (up-down) or shearing (forward back) actions; (iii) changes in geometry, e.g. interlayer contact area; (iv) an increased layer time (UD and FB have longer print times than OR). Still, the combination of these factors did not achieve the same mechanical performance as the nonplanar zigzag toolpath, highlighting the importance of a changed interface between the layers. Apparently, load redistribution had a particularly significant impact on toughness, which increased approximately fourfold between OR-2 and ZZ-4. Unlike planar layers, the zigzag strategy meant loading could not be oriented directly normal to the interface between layers, it is theorised that the zigzag features redistribute some of the load into the filaments, as schematically illustrated in Fig. 9 (a) Schematic of the theorised distribution of load along filaments under tension thanks to zigzag geometry as compared to high stress concentration between layers observed in conventional planar geometry (b)... It is also likely that the bulges identified microscopically (section 3.1.2) had a beneficial impact mechanically in ZZ, UD and FB specimens, as all outperformed the OR specimens (Fig. 8).

These results and analysis indicate that the $Z Z$ specimens have the best mechanical performance of all geometries considered, but further study of this geometry was necessary in order to understand which geometrical features of the zigzag were responsible for the improvement. 


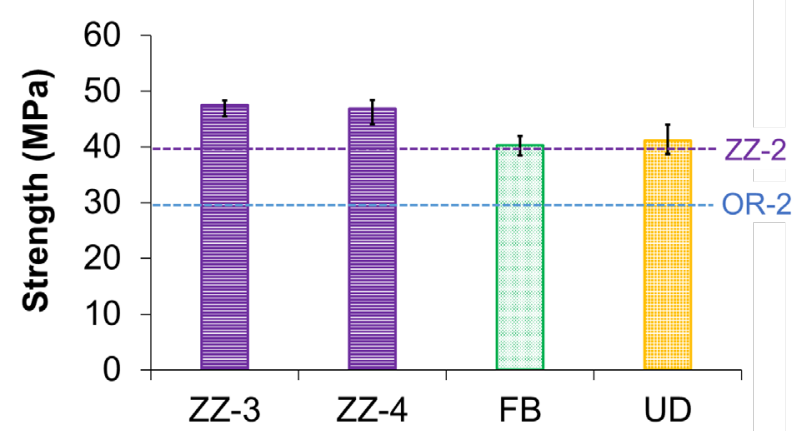

(a)

Specimen type

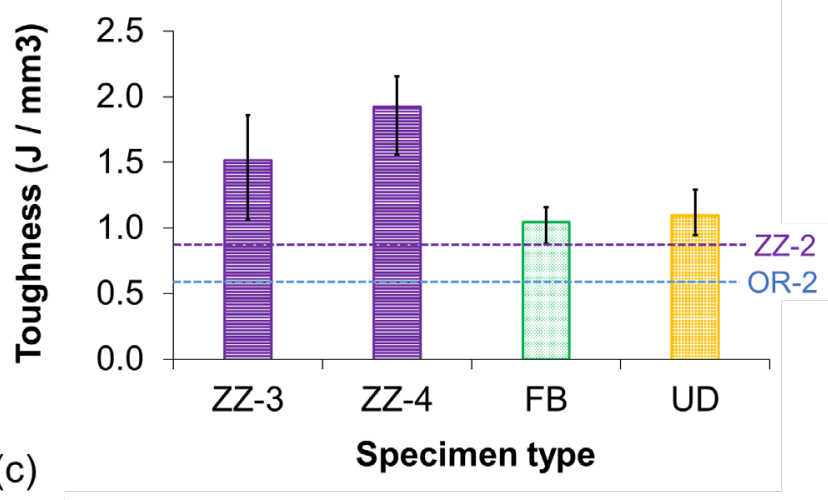

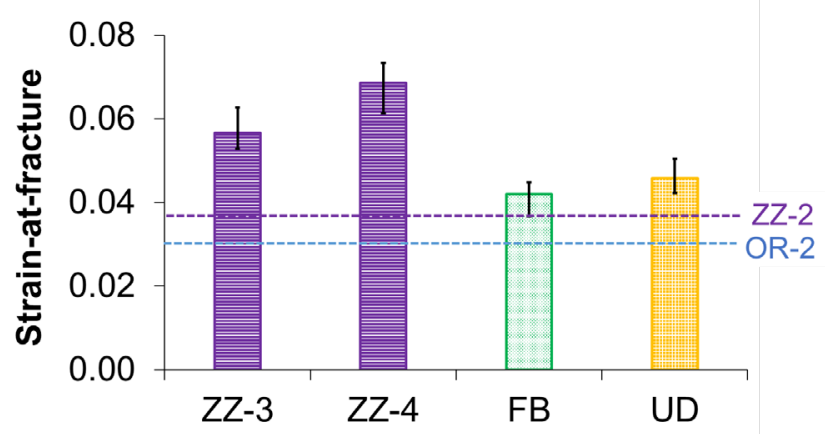

(b)

Fig. 8 Mean effective ultimate tensile strength (a), strain-at-fracture (b) and toughness (c) of ZZ-3, ZZ-4, FB and UD specimens. Error bars indicate the range of results. The labelled dotted lines in each plot indicate the mean performance of ZZ-2 and OR-2 specimens for comparison.
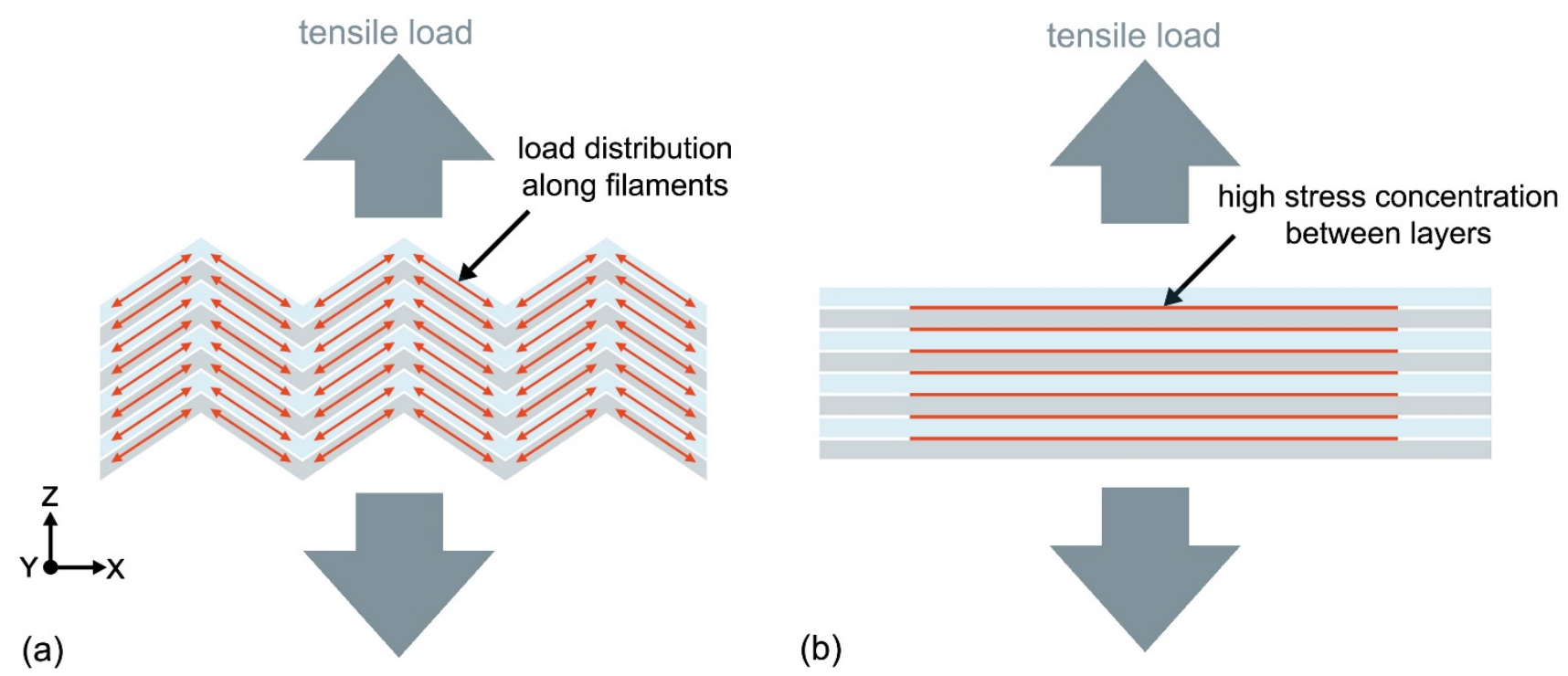

Fig. 9 (a) Schematic of the theorised distribution of load along filaments under tension thanks to zigzag geometry as compared to high stress concentration between layers observed in conventional planar geometry (b). 


\subsubsection{Zigzag-geometry modification}

To assess the impact of zigzag geometry on mechanical performance, a number of modifications were made to the $Z Z$ design by introducing $Z Z_{D S}, Z Z_{D H}$ and $Z Z_{D W}$ specimens (see Fig. 3).

As demonstrated in Fig. 10, modifying the zigzag geometry had very little impact on the effective strength (Fig. 10 (a)), strain-at-fracture (Fig. 10 (b)), or toughness (Fig. 10 (c)) of specimens. Similarly, $Z_{D S}, Z_{D H}$ and $Z Z_{D W}$ demonstrated mechanical performance very close to that of the ZZ-3 specimens, with which they shared comparable mean filament thicknesses.

These results indicate that the specific geometry of the zigzag unit had little influence on the improvement of mechanical performance. It is evident that the range of $Z Z$ unit designs were sufficient to enable similar levels of improved mechanical performance. To further understand the mechanisms influencing the mechanical performance of the studied specimens, fractographic analysis was undertaken. This is the subject of the next section.

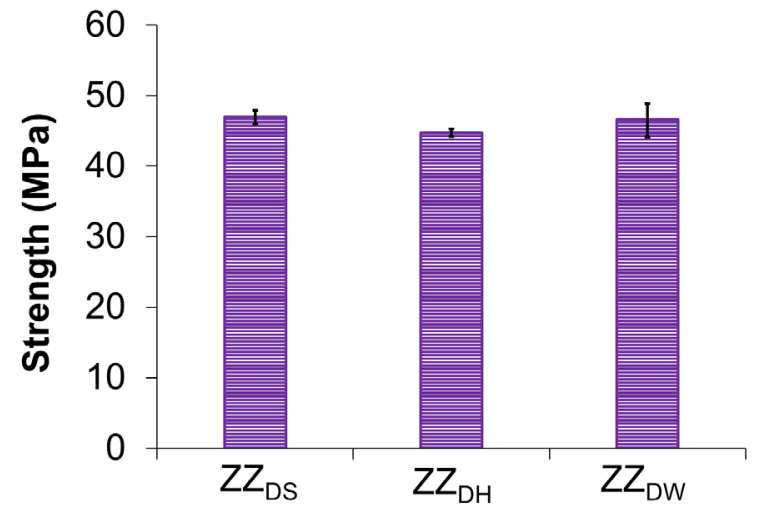

(a)

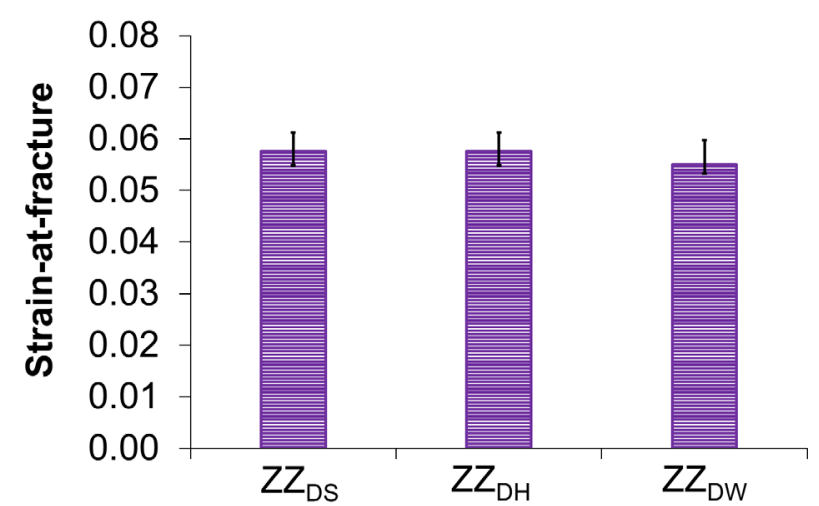

(b)

Specimen Type

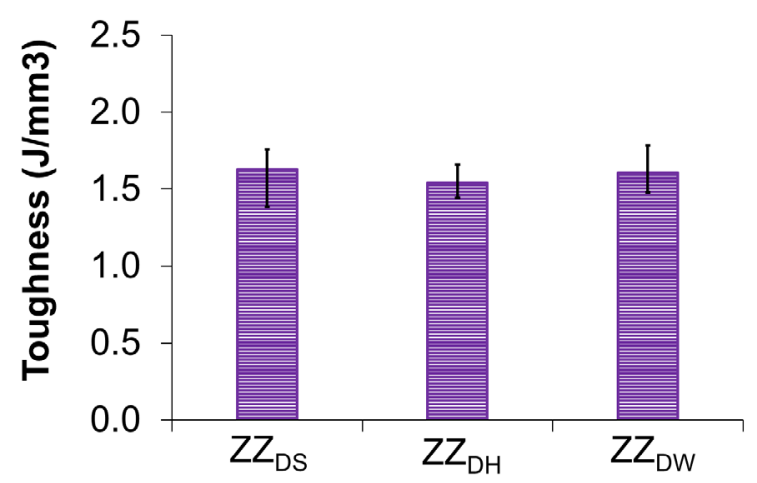

(c)

Specimen Type

Fig. 10 Mean effective ultimate tensile strength (a), strain-at-fracture (b) and toughness (c) of $Z Z_{D S}, Z Z_{D H}$ and $Z Z_{D W}$ specimens. Error bars indicate the range of results. The dashed line indicates the respective level of ZZ-3 specimens. 


\subsection{Fractographic analysis}

Fractography was undertaken to observe fracture occurring at the interface between layers and through filaments in all specimen types (Fig. 11). Through-filament fracture is defined as a fracture which occurs through a filament as opposed to failure following the interfacial groove between filaments (see example of $Z_{\mathrm{DH}}$ specimen (Fig. 12)). The number of through-filament fractures was quantified to assess a fraction of specimens with at least one occurrence of this mechanism (Fig. 13 (a)) together with a mean number of filaments fractured in each specimen (Fig. 13 (b)) for each type.

The conventional specimens OR-1 and OR-2 demonstrated a predominantly interfacial fracture, with the former (with lowest filament thickness) showing only interfacial fracture across all specimens. As the filament thickness was increased, the incidence of throughfilament fractures slightly grew in the OR-2 specimens suggesting the decrease of the effect of stress concentrations caused by the grooves. This demonstrates that even in conventionally printed specimens with sufficient filament thickness, the interface is adequately strong enough to occasionally prompt the fracture path to travel through a filament to another interface (Fig. 13 (a)).

FB specimens largely shared the fracture-path features of OR-1 and OR-2 owing to their planar toolpath. In contrast, the UD specimens demonstrated regular through-filament fracture, suggesting that the up-down motion mixed molten polymer across multiple layers. Most ZZ specimens fractured through filaments. All specimens in ZZ-2, ZZ-4, ZZ and $Z Z_{D W}$ groups fractured through multiple filaments, while $80 \%$ of $Z Z-1$ and $Z Z-3$ specimens demonstrated through-filament fracture. In several $Z_{Z_{D H}}$ specimens each zigzag unit was broken in two places, with the fracture effectively removing the top of each zigzag unit (Fig. 12). As discussed above in reference to Fig. 9 (a), the inclusion of the zigzag deposition likely resulted in redistribution of load along the filaments and thus reduced stress concentration at the interface; so, the fracture more frequently travelled through filaments rather than between them.

Apparently, changes in the fracture path did not necessarily influence the mechanical strength. For example, the FB specimens, comprised of only planar layers, demonstrated very close mechanical strength to that of the ZZ-3 specimens in spite of having very few cases of through-filament fracture (Fig. 11 (a) and (b)). As the extruded filament strength is known to be equivalent to that of bulk material $[11,12]$, this finding supports the findings that the interface material has bulk strength as specimens with through-filament fracture paths and interfacial ones both exhibited comparable mechanical strength. This also indicates that the interfacial grooves between filaments in the ZZ and UD designs did not cause significant enough stress concentration to cause interfacial fracture. The $Z_{D S}, Z_{D H}, Z_{D W}$ and $Z Z-3$ specimens had close mechanical performance, but their numbers of through-filament fractures differed significantly, indicating that the interfacial region provided the same mechanical characteristics as the filament. Still, the character of fracture affected toughness of 3D-printed specimens.

FB specimens showed greater performance in terms of toughness compared to the conventional OR-2 specimens $\left(1.04 \mathrm{~J} / \mathrm{mm}^{3}\right.$ and $\left.0.56 \mathrm{~J} / \mathrm{mm}^{3}\right)$. This could be due to the effect of the microscale bulges observed along the deposited filament (Fig. $5(\mathrm{~h})$ ), which may have reinforced the structure acting as crack arresters and thus improved mechanical performance. This is similar in the UD specimens (Fig. 5 (g)). Importantly, the toughness 
observed in a number of the $\mathrm{ZZ}$ toolpath strategies were significantly greater than those of the conventional specimens. For example, ZZ-4 had toughness nearly four times that of OR$2\left(1.92 \mathrm{~J} / \mathrm{mm}^{3}\right.$ and $0.56 \mathrm{~J} / \mathrm{mm}^{3}$, respectively), correlating with a significantly increased numbers of through-filament fractures in the former specimens. This suggests that the nonplanar interfacial geometry changes resulting from the $Z Z$ strategy may have contributed to their increased toughness by reducing stress concentrations normal to the loading direction typically associated with the interfaces of OR specimens. The next section discusses the advantages, limitations and applications of the findings in this study.

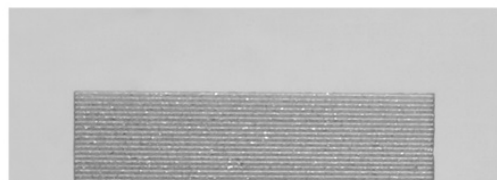

(a)

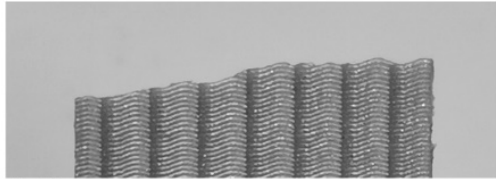

(d)

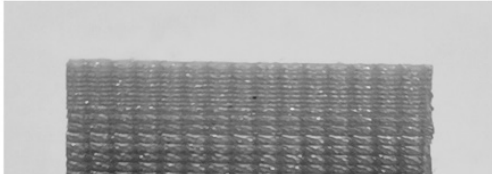

(g)

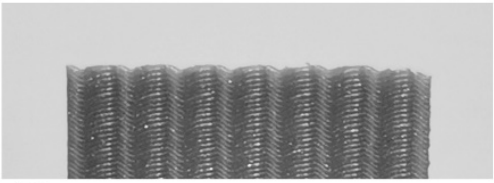

(j)

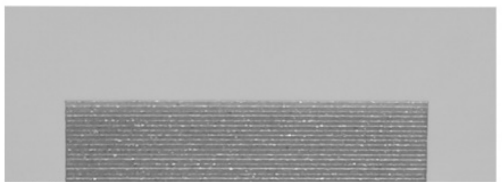

(b)

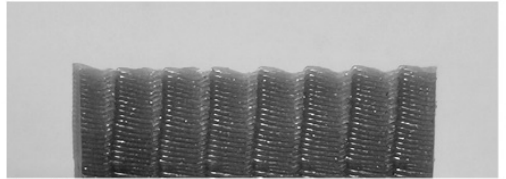

(e)

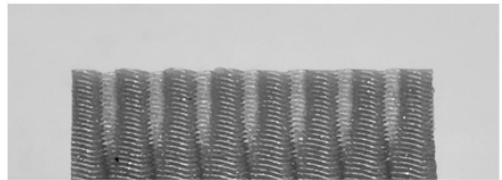

(h)

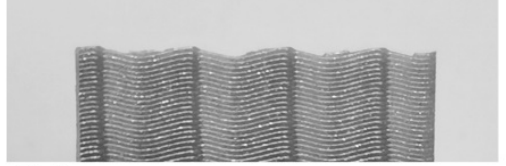

(k)

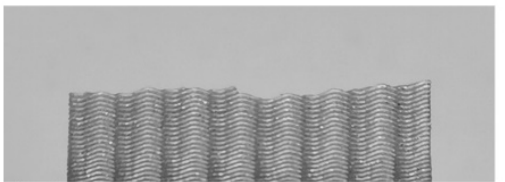

(c)

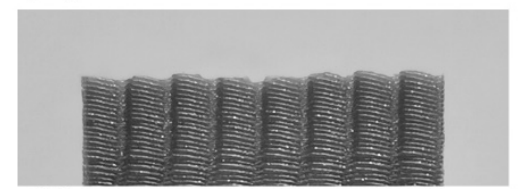

(f)

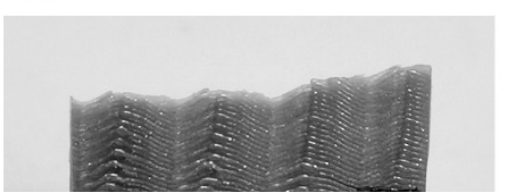

(i)

Fig. 11 Typical fracture paths (side view) after tensile testing: (a) OR-1; (b) OR-2; (c) ZZ-1; (d) ZZ-2; (e) ZZ-3; (f) ZZ-4; (g) FB; (h) UD; (i) ZZZ ; (j) ZZZ $Z$; (k) ZZ $Z Z_{D W}$. 


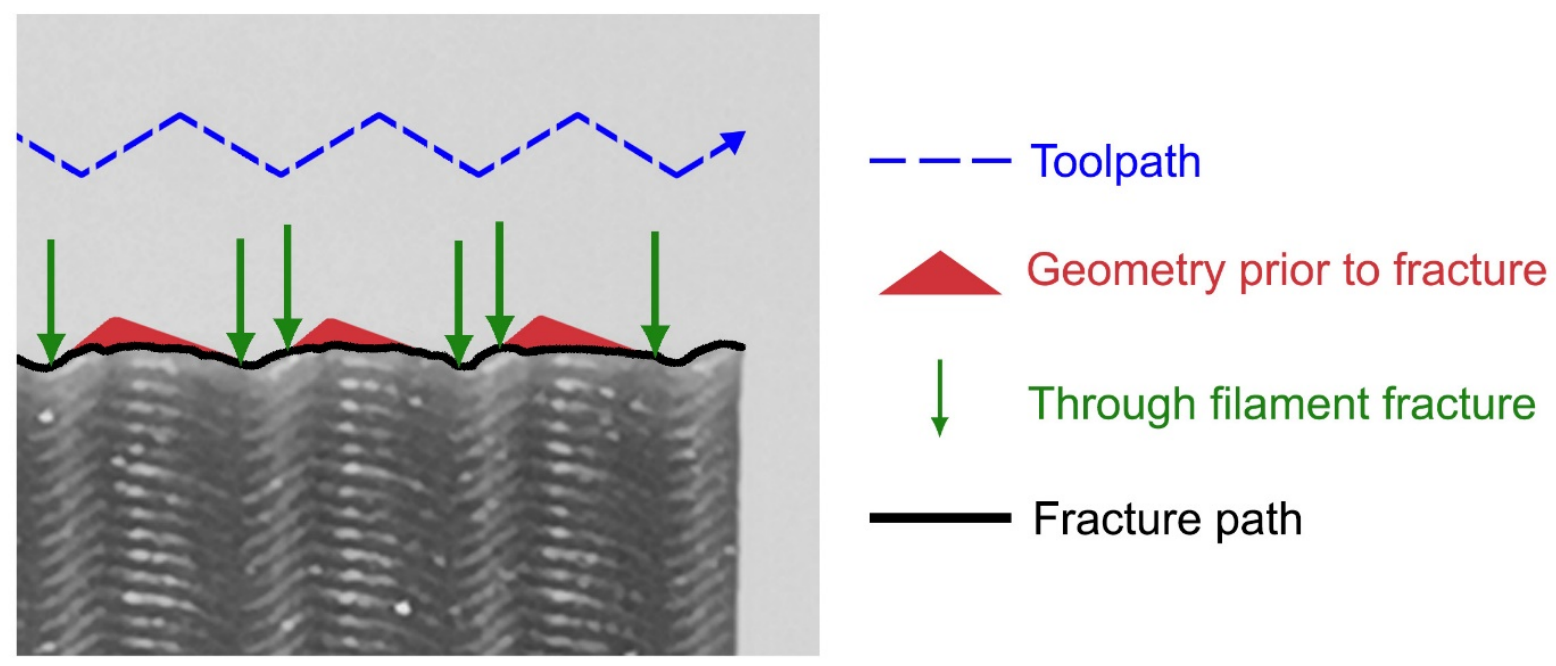

Fig. 12 Typical fracture in $Z Z_{D S}$ specimens with fracture path traveling through multiple filaments. Green arrows indicate the location of the through-filament fractures.

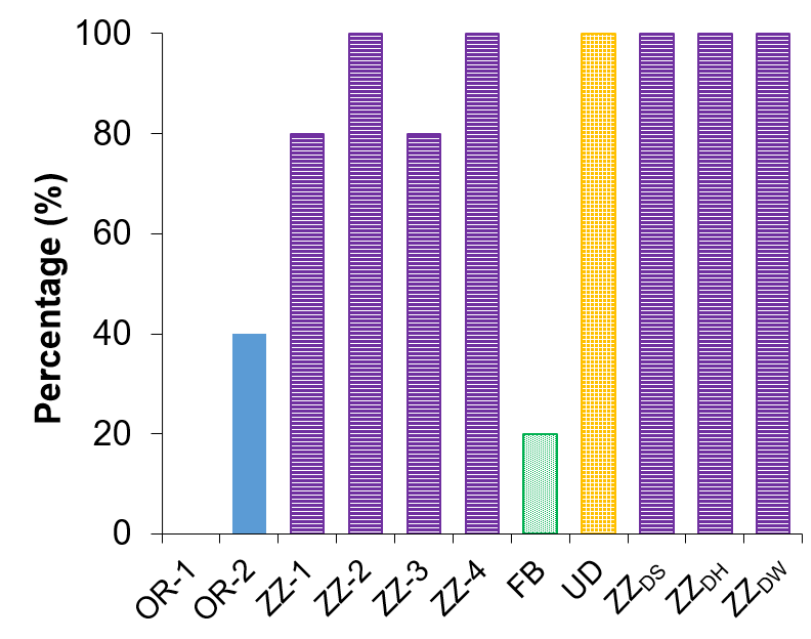

(a)

\section{Specimen Type}

Fig. 13 (a) Fraction of specimens with at least one through-filament fracture. (b) Mean number of through-filament fractures. The error bars indicate the range of through-filament fractures.

\section{Advantages, limitations and applications}

This section discusses the advantages and limitations of the nonplanar techniques developed in this study compared to the existing conventional planar and nonplanar methods of FFF. Additionally, the potential for new nonplanar strategies to be used in a range of end-use parts is considered and demonstrated.

As previously mentioned, the nonplanar strategies suggested in this study are based on repeating nozzle-scale features (with dimensions of approximately $0.5 \mathrm{~mm}$ to $4 \mathrm{~mm}$ ). They 
provide a number of advantages over nonplanar strategies developed in other studies, which utilise large, part-scale curved layers or other techniques. A key advantage of the new methods is the adaptability of the strategy. For example, the designed units are 'modular' and can be repeated numerous times over arbitrary lengths making them suitable for large and small structures. The features developed can provide consistent interfacial mechanical improvements, whereas parts produced with a curved-layer technique can have properties similar to those of OR specimens, but with varying anisotropic directions. Also, the nonplanar strategies developed in this study could be graded and selectively deposited with the mechanical performance of their parts tailored to specific local mechanical requirements. Compared to planar techniques, all ZZ geometries demonstrated mechanical improvement irrespective of the chosen zigzag geometry, provided that the filament geometry (thickness) was sufficient (approximately $0.6 \mathrm{~mm}$ as shown by this study, which represent 3 times the layer height).

Although this study focussed on the mechanical testing of single-filament specimens to enable characterisation at the fundamental scale, a number of additional specimens were developed (using FullControl GCode Designer [29]) and manufactured; they demonstrate the applicability of the suggested zigzag strategy in larger structures (Fig. 14), with ZZ outer and infill walls. Infill structures are typically formed by of a series of interlinked walls, so that mesoscale features, developed in this study, can be used for them. This is a principal difference with the existing nonplanar strategies, as the infill benefits from the capability to grade and adapt the structure selectively by design, additionally the examples demonstrated (Fig. 14) were all designed with toolpath algorithms that avoided collisions between the nozzle and previously deposited material, illustrating the capability to use similar algorithms for the use of zigzag structures in more complex geometries. Similarly, for applications where the selection of a specific outer-wall print strategy is critical for aesthetic (or other) reasons, the use of a zigzag infill with an outer skin comprised of an alternative printing strategy is feasible, with the resultant part still benefiting mechanically from the internal $Z Z$ infill strategy. An example of such a part with zigzag infill walls but planar outer walls is shown in Fig. 14 (b). The design freedom afforded by FullControl GCode Designer software [29] allows the development and evaluation of more complex structures with zigzag infill geometries. Furthermore, dedicated scripts could be written to use the zigzag strategy for arbitrary shapes and preloaded models. Changes in toolpaths resulted in significant variation in surface texture, indicating that the use of unconventional planar and nonplanar schemes could also be utilised to generate surface finish for specific applications. It is conceivable that these could be beneficial e.g. for biological cellular growth, increased friction or enhanced opticals. Several examples of the surface textures generated in this study are shown in Fig. 15. A gradual transition from planar strategies on top/bottom surfaces of a part to a nonplanar internal structure are also possible, to optimise mechanical performance and density.

The distinct mechanical advantages provided by the nonplanar toolpaths developed in this study indicate that the strategies could have a broad applicability in FFF, particularly where improved mechanical performance of interlayers is desirable or necessary. The plot in Fig. 16 demonstrates the mechanical advantage of utilising ZigZagZ toolpaths over the conventional planar strategy. The range of ZigZagZ values are based on ZZ-2 and ZZ-3 specimens because OR-2 specimens had a mean thickness in-between them. For all properties, the ZigZagZ approach demonstrated superior mechanical performance to the conventional specimens, even when specimens were lower in weight. 
The implementation of $Z Z$ for infill indicates that nonplanar strategy should be considered as a complete or partial replacement of planar ones. The mesoscale features make this applicable to a vast range of parts, from high-precision medical devices with single-filament walls to large structural parts with infill e.g. for automotive parts. Among other potential applications are concrete printing for construction of buildings and other types of 3D printing, where interfaces reduce mechanical performance. Importantly, these designs could be implemented into 3D printing software in a relatively straightforward manner.

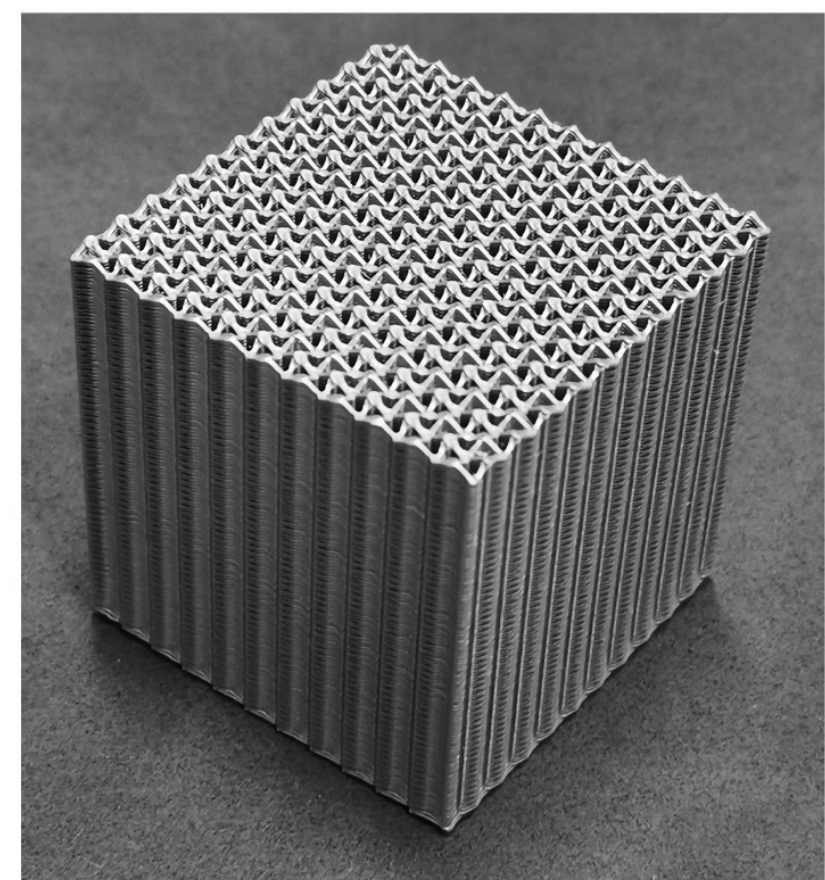

(a)

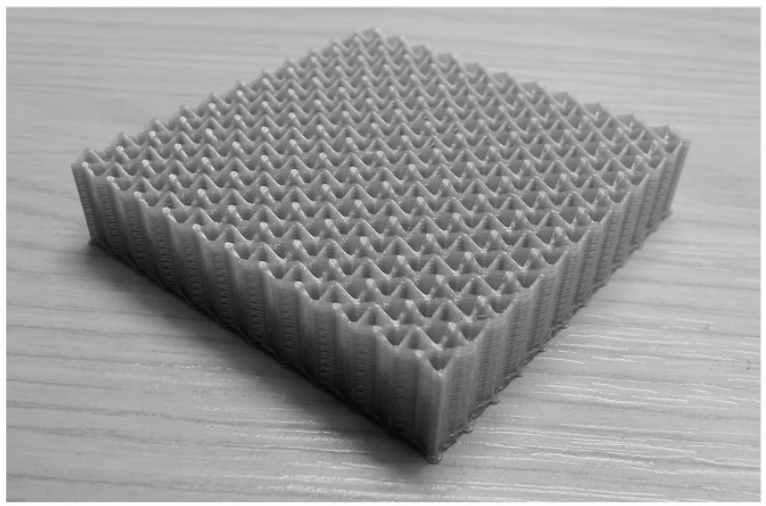

(c)

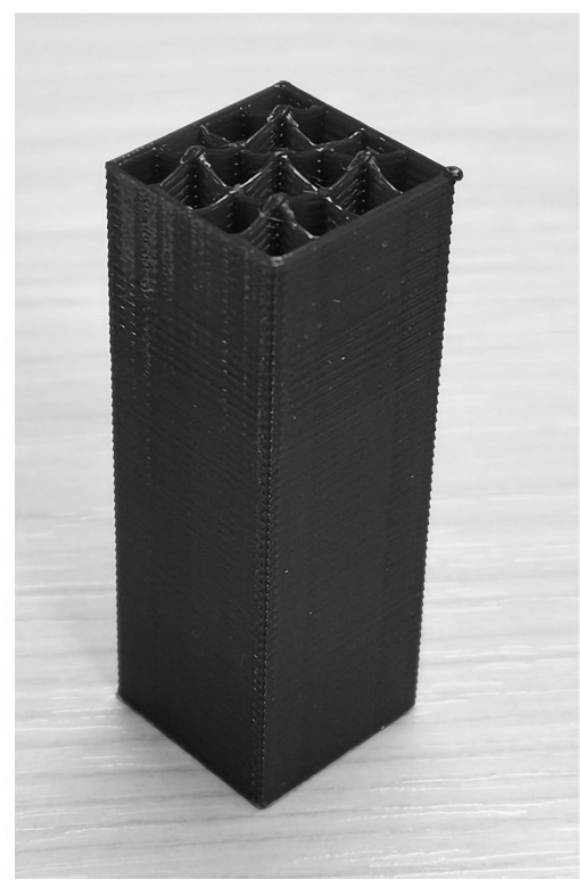

(b)

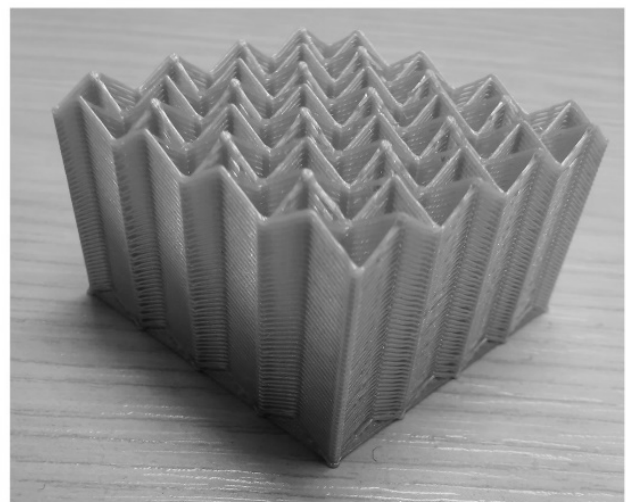

(d)

Fig. 14 Parts manufactured using the zigzag toolpath geometry developed in this study with both the infill and outer walls formed by zigzag-deposited filaments. The variation in number of infill filaments and the grid density of the structures is demonstrated, with relatively large numbers of infill filaments with high density in (a) and (c), and fewer filaments with lower grid density in (b) and (d). 


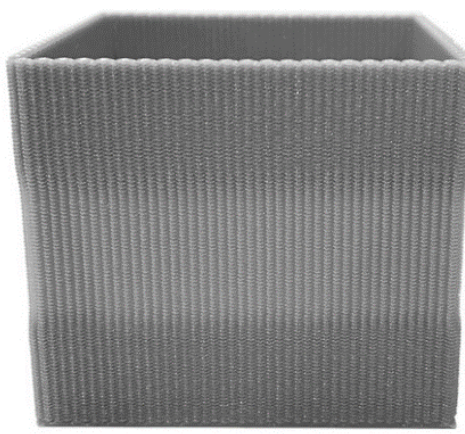

(a)

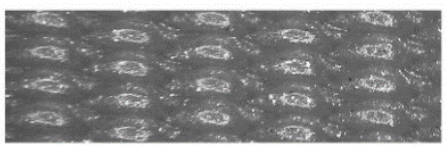

(b)

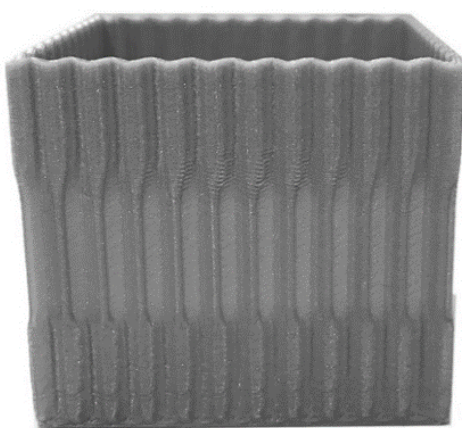

(c)

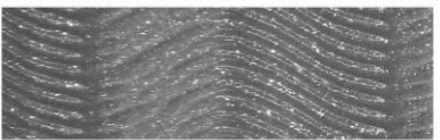

(d)

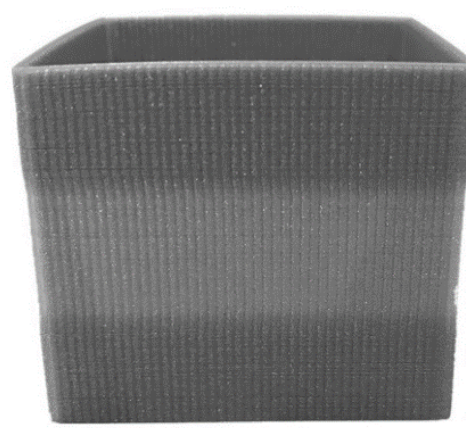

(e)

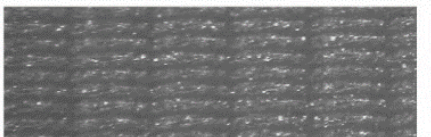

(f)

Fig. 15 Surface texture of three specimen types: (a) and (b) UD unit design, with units offset by half a unit; (c) and (d) ZZZ $Z Z_{D S}$ geometry; (e) and (f) FB geometry. ((b), (d) and (f) are closeups for respective specimens).

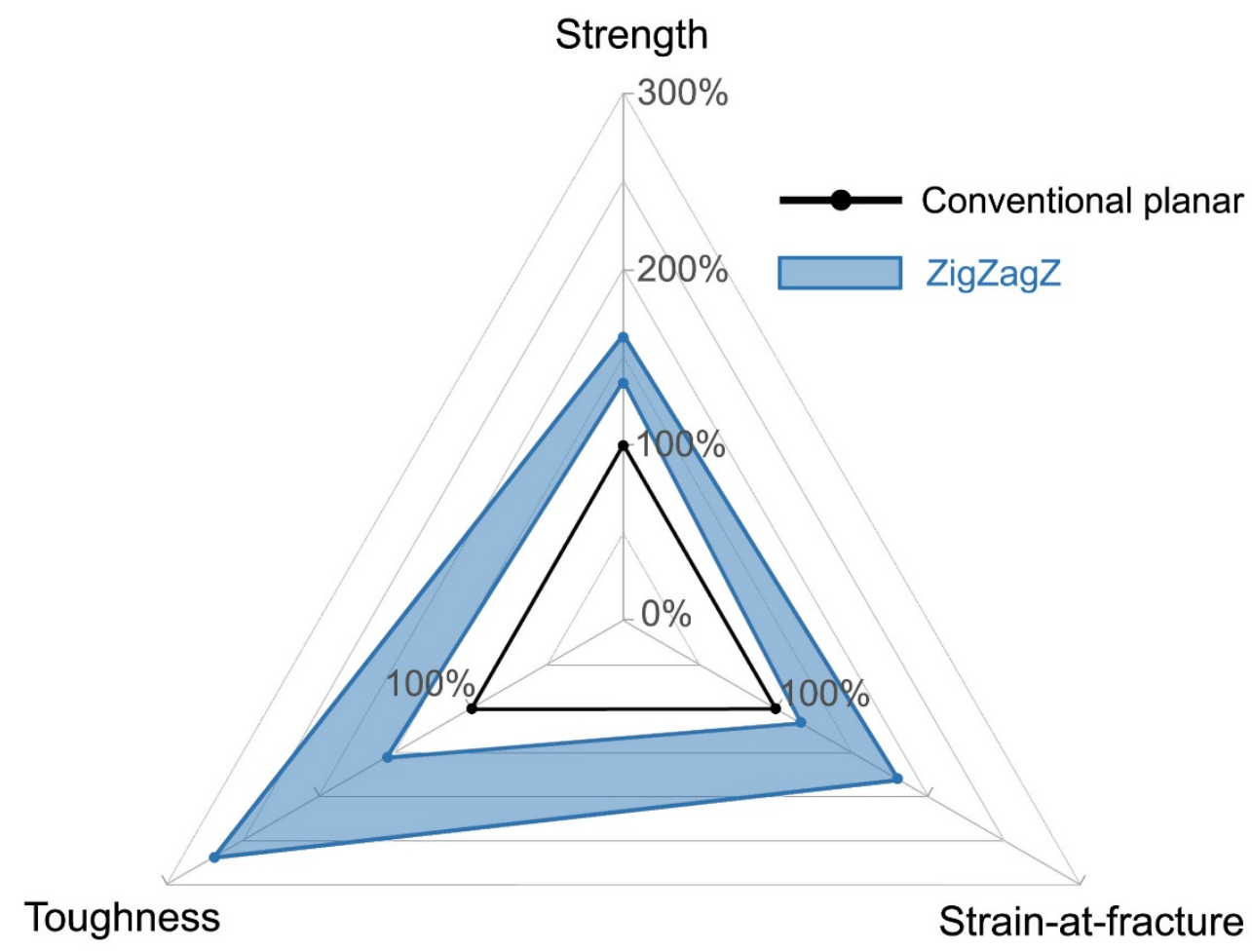

Fig. 16 Comparison of normalised mean mechanical performance of the conventional printing strategy and the ZigZagZ (for a range of two specimen weights that encompass the weight of the conventional planar specimen). 


\section{Conclusions and future work}

This study identified that the use of the developed ZigZagZ toolpath geometries in FFF can provide significant mechanical improvement in specimens loaded normal to the direction of filaments ( $Z$ direction) compared to conventional planar methods. The influence of the toolpaths on extruded filament geometry was analysed microscopically to provide a new understanding of the effects of deposition on resultant filament-scale geometry. The strength, strain-at-fracture and toughness of the optimal zigzag specimen were significantly improved. Analysis showed that the nonplanar deposition in the specimens resulted in increased occurrence of through-filament fracture, demonstrating that the new geometry was sufficient to redirect (into the filament) loads that are typically concentrated at the interface; this led to improved toughness. The geometries developed in this study were formed by repeating nozzle-scale units that are widely applicable to a vast range of features including infill, outer walls and single-filament structures and can be used in different fields including construction. The manufacturing strategies developed in this study are suitable for manufacture of end-use parts, where interlayer mechanical performance is of critical importance.

The future work in this area should consider development and integration of the zigzag geometry method into other 3D printed structures, such as multi-filament walls. The authors are currently investigating the use of $Z Z$ in infill structures with varying angles to investigate the mechanical capabilities for use in infill structures. Additionally, investigation of the impact of $Z Z$ on the filament-direction loading could be considered along with additional loading mechanisms, such as compression testing and 3-point bending analysis. Identifying a means of integrating the $\mathrm{ZZ}$ geometry into conventional printing software would be beneficial for end use parts particularly.

\section{Acknowledgements}

This research did not receive any specific grant from funding agencies in the public, commercial, or not-for-profit sectors.

\section{References}

[1] S. Ahn, M. Montero, D. Odell, S. Roundy, P.K. Wright, Anisotropic material properties of fused deposition modeling ABS, Rapid Prototyp. J. 8 (2002) 248-257. https://doi.org/10.1108/13552540210441166.

[2] O.S. Es-Said, J. Foyos, R. Noorani, M. Mendelson, R. Marloth, B.A. Pregger, Effect of layer orientation on mechanical properties of rapid prototyped samples, Mater. Manuf. Process. 15 (2000) 107-122. https://doi.org/10.1080/10426910008912976.

[3] A.K. Sood, R.K. Ohdar, S.S. Mahapatra, Improving dimensional accuracy of Fused Deposition Modelling processed part using grey Taguchi method, 2009. https://doi.org/10.1016/j.matdes.2009.04.030.

[4] I. Durgun, R. Ertan, Experimental investigation of FDM process for improvement of mechanical properties and production cost, Rapid Prototyp. J. 20 (2014) 228-235. https://doi.org/10.1108/RPJ-10-2012-0091. 
[5] J.P. Thomas, J.F. Rodríguez, J.E. Renaud, Mechanical behavior of acrylonitrile butadiene styrene (ABS) fused deposition materials. Experimental investigation, Rapid Prototyp. J. 7 (2001) 148-158. https://doi.org/10.1108/13552540110395547.

[6] J.C. Riddick, M.A. Haile, R. Von Wahlde, D.P. Cole, O. Bamiduro, T.E. Johnson, Fractographic analysis of tensile failure of acrylonitrile-butadiene-styrene fabricated by fused deposition modeling, Addit. Manuf. 11 (2016) 49-59. https://doi.org/10.1016/j.addma.2016.03.007.

[7] V.E. Kuznetsov, A.N. Solonin, O.D. Urzhumtsev, R. Schilling, A.G. Tavitov, Strength of PLA components fabricated with fused deposition technology using a desktop 3D printer as a function of geometrical parameters of the process, Polymers (Basel). 10 (2018). https://doi.org/10.3390/polym10030313.

[8] C. Koch, L. Van Hulle, N. Rudolph, Investigation of mechanical anisotropy of the fused filament fabrication process via customized tool path generation, Addit. Manuf. 16 (2017) 138-145. https://doi.org/10.1016/j.addma.2017.06.003.

[9] S. Ziemian, M. Okwara, C.W. Ziemian, Tensile and fatigue behavior of layered acrylonitrile butadiene styrene, Rapid Prototyp. J. 21 (2015) 270-278. https://doi.org/10.1108/RPJ-09-2013-0086.

[10] T.J. Coogan, Kazmer, Bond and part strength in fused deposition modeling, Rapid Prototyp. J. 23 (2017) 414-422. https://doi.org/10.1108/RPJ-03-2016-0050.

[11] J. Allum, A. Gleadall, V. Silberschmidt, Fracture of 3D-printed polymers: crucial role of filament-scale geometric features (submitted for publication), Eng. Fract. Mech. 224 (2020) 106818. https://doi.org/10.1016/j.engfracmech.2019.106818.

[12] J. Allum, A. Moetazedian, A. Gleadall, V. V. Silberschmidt, Interlayer bonding has bulk-material strength in extrusion additive manufacturing: new understanding of anisotropy, Addit. Manuf. (2020) 101297.

https://doi.org/10.1016/j.addma.2020.101297.

[13] M. Spoerk, F. Arbeiter, H. Cajner, J. Sapkota, C. Holzer, Parametric optimization of intra- and inter-layer strengths in parts produced by extrusion-based additive manufacturing of poly(lactic acid), J. Appl. Polym. Sci. 134 (2017). https://doi.org/10.1002/app.45401.

[14] B. V. Reddy, N. V. Reddy, A. Ghosh, Fused deposition modelling using direct extrusion, Virtual Phys. Prototyp. 2 (2007) 51-60. https://doi.org/10.1080/17452750701336486.

[15] C. Bellehumeur, L. Li, Modeling of bond formation between polymer filaments in the Fused Deposition Modeling process, J. Manuf. Process. 6 (2004) 170-178. https://doi.org/10.1016/S1526-6125(04)70071-7.

[16] N. Aliheidari, J. Christ, R. Tripuraneni, S. Nadimpalli, A. Ameli, Interlayer adhesion and fracture resistance of polymers printed through melt extrusion additive manufacturing process, Mater. Des. 156 (2018) 351-361. https://doi.org/10.1016/j.matdes.2018.07.001.

[17] F. Arbeiter, M. Spoerk, J. Wiener, A. Gosch, G. Pinter, Fracture mechanical characterization and lifetime estimation of near-homogeneous components produced by fused filament fabrication, Polym. Test. 66 (2018) 105-113. https://doi.org/10.1016/j.polymertesting.2018.01.002.

[18] C. Duty, J. Failla, S. Kim, T. Smith, J. Lindahl, V. Kunc, Z-Pinning approach for 3D printing mechanically isotropic materials, Addit. Manuf. 27 (2019) 175-184. 
https://doi.org/10.1016/j.addma.2019.03.007.

[19] B. Huang, S.B. Singamneni, Curved layer adaptive slicing (CLAS) for fused deposition modelling, Rapid Prototyp. J. 21 (2015) 354-367. https://doi.org/10.1108/RPJ-062013-0059.

[20] S. Ravindrababu, Y. Govdeli, Z.W. Wong, E. Kayacan, Evaluation of the influence of build and print orientations of unmanned aerial vehicle parts fabricated using fused deposition modeling process, J. Manuf. Process. 34 (2018) 659-666.

https://doi.org/10.1016/j.jmapro.2018.07.007.

[21] F. Rosa, S. Graziosi, A parametric and adaptive slicing (PAS) technique: general method and experimental validation, Rapid Prototyp. J. 25 (2019) 126-142. https://doi.org/10.1108/RPJ-11-2016-0184.

[22] B. Huang, S. Singamneni, Curved layer fused deposition modeling with varying raster orientations, Appl. Mech. Mater. 446-447 (2014) 263-269.

https://doi.org/10.4028/www.scientific.net/AMM.446-447.263.

[23] L. Chen, M.F. Chung, Y. Tian, A. Joneja, K. Tang, Variable-depth curved layer fused deposition modeling of thin-shells, Robot. Comput. Integr. Manuf. 57 (2019) 422-434. https://doi.org/10.1016/j.rcim.2018.12.016.

[24] K. Xu, Y. Li, L. Chen, K. Tang, Curved layer based process planning for multi-axis volume printing of freeform parts, CAD Comput. Aided Des. 114 (2019) 51-63. https://doi.org/10.1016/j.cad.2019.05.007.

[25] A. V. Shembekar, Y.J. Yoon, A. Kanyuck, S.K. Gupta, Generating robot trajectories for conformal three-dimensional printing using nonplanar layers, J. Comput. Inf. Sci. Eng. 19 (2019) 1-13. https://doi.org/10.1115/1.4043013.

[26] A.T. Alsharhan, T. Centea, S.K. Gupta, Enhancing mechanical properties of thinwalled structures using non-planar extrusion based additive manufacturing, ASME 2017 12th Int. Manuf. Sci. Eng. Conf. MSEC 2017 Collocated with JSME/ASME 2017 6th Int. Conf. Mater. Process. 2 (2017) 1-9. https://doi.org/10.1115/MSEC2017-2978.

[27] S. Singamneni, A. Roychoudhury, O. Diegel, B. Huang, Modeling and evaluation of curved layer fused deposition, J. Mater. Process. Technol. 212 (2012) 27-35. https://doi.org/10.1016/j.jmatprotec.2011.08.001.

[28] A. Moetazedian, A. Gleadall, X. Han, V. V. Silberschmidt, Effect of environment on mechanical properties of 3D printed polylactide for biomedical applications, J. Mech. Behav. Biomed. Mater. 102 (2020) 103510. https://doi.org/10.1016/j.jmbbm.2019.103510.

[29] A. Gleadall, FullControl GCode Designer: open source software for unconstrained design in additive manufacturing. Submitted for publication. Software available at: www.fullcontrolgcode.com., (2020) 1-24.

[30] ASTM International, D1708-18 Standard Test Method for Tensile Properties of Plastics by Use of Microtensile Specimens, ASTM International, West Conshohocken, PA, 2018, https://doi.org/10.1520/D1708-18.

[31] L. D'Urso, M.R. Acocella, G. Guerra, V. lozzino, F. De Santis, R. Pantani, PLA melt stabilization by high-surface-area graphite and carbon black, Polymers (Basel). 10 (2018) 1-13. https://doi.org/10.3390/polym10020139.

[32] J. Allum, A. Moetazedian, A. Gleadall, V. V. Silberschmidt, Interlayer bonding has bulk-material strength in extrusion additive manufacturing: new understanding of anisotropy, Addit. Manuf. 34 (2020) 101297. 
https://doi.org/10.1016/j.addma.2020.101297.

[33] A. Moetazedian, A.S. Budisuharto, V.V. Silberschmidt, A. Gleadall, CONVEX (CONtinuously Varied EXtrusion): a new scale of design for additive manufacturing, Addit. Manuf. Accepted P (2020).

[34] Ultimaker, Technical Data Sheet PLA, (2017) 1-3. http://www.farnell.com/datasheets/2310522.pdf. 